\title{
Attention improves information flow between neuronal populations without changing the communication subspace
}

Authors: Ramanujan Srinath, Douglas A. Ruff, Marlene R. Cohen

Lead Contact: Ramanujan Srinath

Affiliations: Department of Neuroscience and Center for the Neural Basis of Cognition,

University of Pittsburgh, Pittsburgh, PA, USA

Email: ramanujan@pitt.edu,ruffd@pitt.edu,cohenm@pitt.edu 


\section{Summary}

2 Visual attention allows observers to flexibly use or ignore visual information, suggesting that

3 information can be flexibly routed between visual cortex and neurons involved in decision-

4 making. We investigated the neural substrate of flexible information routing by analyzing the

5 activity of populations of visual neurons in the medial temporal area (MT) and oculomotor

6 neurons in the superior colliculus (SC) while rhesus monkeys switched spatial attention. We

7 demonstrated that attention increases the efficacy of visuomotor communication: trial-to-trial

8 variability of the population of SC neurons was better predicted by the activity of MT neurons

9 (and vice versa) when attention was directed toward their joint receptive fields. Surprisingly, this

10 improvement in prediction was not explained or accompanied by changes in the dimensionality

11 of the shared subspace or in local or shared pairwise noise correlations. These results suggest a

12 mechanism by which visual attention can affect perceptual decision-making without altering

13 local neuronal representations. 


\section{Introduction}

16 Perhaps the most impressive hallmark of the nervous system is its flexibility. We effortlessly

17 alternate between relying on or ignoring the same sensory information in different contexts.

18 Visual attention dramatically affects perception and a wide variety of measures of neural activity

19 in essentially every visual and visuomotor brain area (for reviews, see (Maunsell, 2015; Moore

20 and Zirnsak, 2017)). Attention flexibly modulates signatures of neuronal activity including trial-

21 averaged firing rates (Desimone and Duncan, 1995; Maunsell, 2015; Reynolds and Chelazzi,

22 2004), shared variability between pairs of neurons in the same (Cohen and Maunsell, 2009,

23 2011; Gregoriou et al., 2014; Herrero et al., 2013; Luo and Maunsell, 2015; Mayo and Maunsell,

24 2016; Mitchell et al., 2009; Nandy et al., 2017; Ni et al., 2018; Ruff and Cohen, 2014a, 2014b,

25 2016a, 2019; Verhoef and Maunsell, 2017; Yan et al., 2014; Zénon and Krauzlis, 2012) and

26 different brain areas (Oemisch et al., 2015; Pooresmaeili et al., 2014; Ruff and Cohen, 2016a;

27 Ruff et al., 2016), interdependence of neuronal populations on a range of timescales (Azouz and

28 Gray, 2003; Bichot et al., 2005; Bosman et al., 2012; Briggs et al., 2013; Buffalo et al., 2011;

29 Buschman and Miller, 2007; Dagnino et al., 2014; Fries, 2015; Fries et al., 2001; Gregoriou et

30 al., 2009; Klink et al., 2017; Lakatos et al., 2008; Miller and Buschman, 2013; Moore and

31 Armstrong, 2003; Ruff and Cohen, 2016a, 2017; Saalmann et al., 2007; Salinas and Sejnowski,

32 2001; Saproo and Serences, 2014; Womelsdorf and Fries, 2007; Womelsdorf et al., 2006a), and

33 the dimensionality of population activity within each brain area (Cowley et al., 2020; Huang et

34 al., 2019; Ruff et al., 2020).

The behavioral effects of attention make it clear that visual information can be flexibly routed: a stimulus can either guide or be unrelated to a perceptual decision depending on the task condition (Carrasco, 2011; Egeth and Yantis, 1997; Kohn et al., 2016a; Maunsell, 2015). In the visual system, neurons in each area send projections to a variety of different sensory, association,

40 and motor areas, and only a small proportion of neuronal population activity is shared between

41 even highly connected brain areas (Semedo et al., 2019). Recent work used correlative methods

42 to identify a functional 'communication subspace', which consists of the dimensions of neuronal

43 population space in which trial-to-trial variability is shared between areas (Semedo et al., 2019,

44 2020). We similarly adopt the term 'communication' to refer to functional communication (i.e.,

45 shared trial-to-trial variability in responses to the same visual stimulus). 
47 An exciting possibility is that modulations in the shape or the constitution of this subspace could be a substrate for flexible, attention-dependent routing of sensory information. Compared to its behavioral effects, attention has remarkably modest effects on the amount of visual information encoded in visual cortex (Ruff and Cohen, 2019). Instantiating task or attentional flexibility via flexible routing rather than information coding could allow the brain to retain irrelevant visual information for future behavior or memory while the most relevant visual information guides

53 behavior.

We investigated three potential mechanisms of flexible information flow between visual cortex and premotor neurons involved in decision-making. We tested the hypotheses that attention modulates information flow between areas by (1) changing the way visual or task information is represented in a local population, (2) changing the communication subspace itself, and/or (3) changing the efficacy of information transfer (Figure 1d).

Our strategy was to analyze functional communication between neuronal populations in visual and premotor areas while animals switched attention toward or away from their joint receptive fields. We recorded simultaneously from dozens of visual neurons in the medial temporal area (MT) and oculomotor neurons in the superior colliculus (SC) with overlapping receptive fields while rhesus monkeys performed a task in which they switched spatial attention, alternatingly using or ignoring the stimulus in the joint receptive fields of the recorded neurons. We used recently published methods for analyzing functional relationships between populations of neurons by assessing the dimensionality of shared variability and the extent to which the activity of one population could be predicted by the other (Semedo et al., 2019, 2020). We focused on trial-to-trial fluctuations in responses to the same visual stimulus because these are related to

71 functional connectivity rather than simply reflecting tuning for similar stimuli (for review, see

72 (Cohen and Kohn, 2011; Umakantha et al., 2020)), and have been shown to be correlated with

73 choice behavior (Ni et al., 2018; Ruff et al., 2018).

75 We found strong evidence for our third hypothesis, that attention improves the efficacy of

76 functional communication between visual and premotor neurons. Trial-to-trial variability of the 
77 population of SC neurons was better predicted by the activity of MT neurons (and vice versa)

78 when attention was directed inside their joint receptive fields. This enhanced functional

79 communication was not explained by increases or decreases in private or shared pairwise noise

80 variability or a change in the number of private or shared dimensions of neuronal population

81 activity.

83 This enhanced functional communication was not restricted to interactions between visual and

84 premotor neurons. An independent dataset of simultaneously recorded neurons in primary visual

85 cortex (V1) and in MT revealed that attention also increases functional communication within

86 visual cortex-Even though the attention-related change in pairwise correlations and response

87 dimensionality within V1 was small compared to MT or SC, attention significantly enhanced our

88 ability to predict the responses of single MT neurons from population activity in V1. Similarly,

89 the effects of attention on functional communication were similar between MT and visual or

90 motor neurons in the SC.

92 Our study provides a blueprint for combining behavioral paradigms that vary cognitive processes

93 with dimensionality reduction and regression analyses to study how information can be flexibly

94 routed in the nervous system. We used these methods to demonstrate that attention substantially

95 improves the prediction performance between areas, more faithfully communicating information

96 about attended stimuli, independent of changes in pairwise correlations or the dimensionality of

97 either the local population or the shared variability. These results are the first demonstration of

98 how attention affects the activity of distinct but connected populations of neurons in a way that

99 affects the functional communication of visual information. They suggest a mechanism by which

100 cognitive processes can affect perceptual decision making in ways that are independent of

101 changes to the local neuronal representations.

102

\section{Results}

104 We compared evidence consistent with several potential mechanisms for flexible routing of

105 information. We chose a widely studied cued direction change detection task to study the

106 behavioral effects of attention on visual perception, and three brain regions that are known to

107 contribute to motion perception and visually-guided decision making - primary visual cortex 
108 (V1), the middle temporal area (MT), and the superior colliculus (SC). While rhesus monkeys

109 performed the motion change detection task (Figure 1a), we recorded simultaneously from either

110 dozens of neurons in MT and SC (Figure 1b) with overlapping receptive fields (Figure 1c;

111 different aspects of these data were previously reported in Ruff and Cohen, 2019), or from

112 several dozen neurons in V1 and a single MT neuron (Figure 6; different aspects of these data

113 were previously reported in Ruff and Cohen, 2016a, 2016b). During the simultaneous MT-SC

114 recordings, the monkey was cued as to which of two stimuli was most likely to change during a

115 block of trials. This cued stimulus was placed either inside the overlapping receptive fields (RFs)

116 of the recorded MT and SC neurons or in the opposite hemifield (Figure 1c). Throughout this

117 manuscript, attend in refers to the trials where attention was directed toward the joint RFs and

118 attend out refers to trials where attention was directed to the opposite hemifield. The monkey

119 was rewarded for making a saccade to the location of the direction change, which occurred at a

120 random and unsignaled time. The ability of the animal to detect the median difficulty changes in

121 grating direction is enhanced by $\sim 25 \%$ on average across sessions when attention was directed

122 to the location of the change (cued 76.5\% detected, uncued 51.8\% detected) (Ruff and Cohen,

123 2019). We analyzed the spike counts of each visually responsive multi-unit recorded from MT

124 and SC during presentations of identical Gabor stimuli before the direction change (excluding

125 the first presentation in each trial to remove adaptation effects). We also analyzed spike counts of

126 each SC unit with elevated firing rates before saccade onset to the contralateral visual field. In

127 the V1-MT data set, we tested our hypotheses on the responses of groups of V1 neurons whose

128 receptive fields overlapped either of two small stimuli, both of which were inside the RF of the

129 MT neuron (Ruff and Cohen, 2016a, 2016b).

131 Signatures of population interactions that underlie attentional mechanisms

132 We tested the following non-mutually exclusive hypotheses (schematized in Figure 1d) about

133 how attention might modulate information flow within and between areas. (a) Attention

134 primarily modulates communication between areas by changing the dimensionality of either the

135 private or the shared subspace (Figure 1d, left column). (b) Attention improves the fidelity of

136 communication within local populations; this would be observable as an improvement in the

137 ability to predict the activity of one subset of a neurons in a population from the activity of a

138 different subset of neurons in the same area (Figure 1d, middle column) (c) Attention improves 
139 the fidelity of communication across brain regions; this would be evident in the improved

140 accuracy of prediction of neural activity of one region using the activity of the other and vice

141 versa (Figure 1d right column).

\section{Figure 1:}

(0.75-page width - 1.5 column)

a

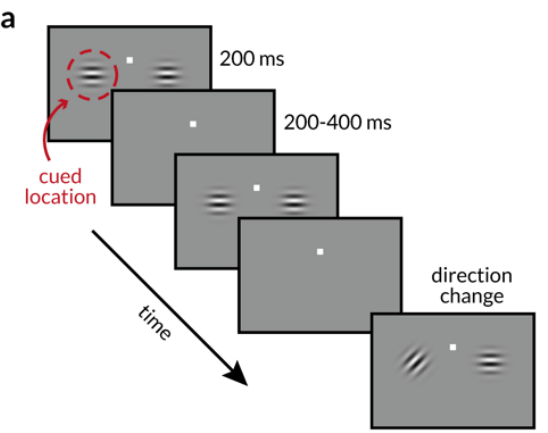

C

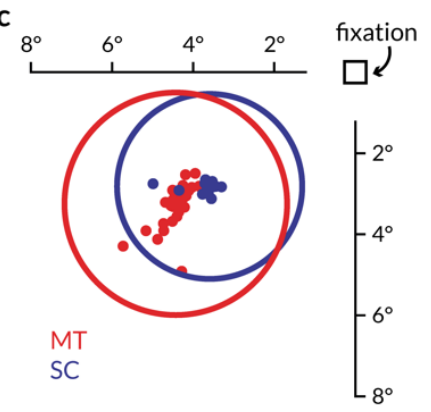

b

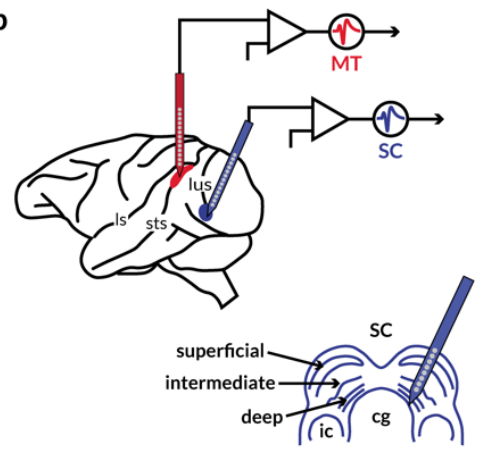

d

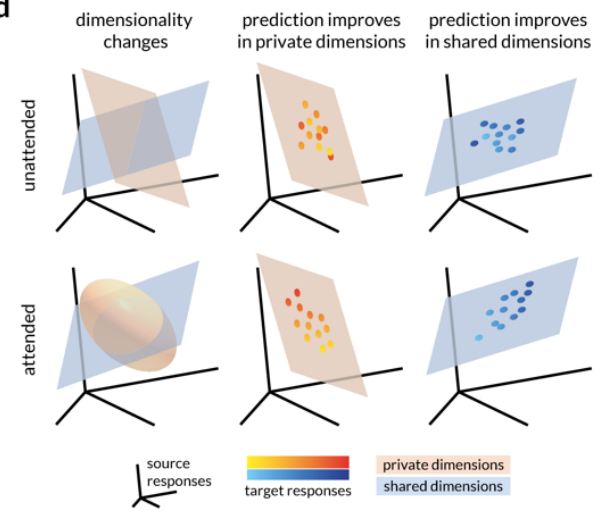

Behavioral task, recording sites, receptive fields, and schematic of hypotheses.

a: Schematic of the motion direction change detection task. The monkeys were cued in blocks of trials to expect changes in motion direction at one of two spatial locations (cue was $80 \%$ valid). The monkey started the trial by fixating a central spot. Two small Gabor stimuli

synchronously flashed on for 200ms and off for a randomized period of 200-400ms. One of the stimuli was positioned inside the joint receptive fields of the MT and SC neurons, and the other was placed in the opposite hemifield. Both stimuli moved in a direction that was chosen to drive the MT population well. After a randomized number of stimulus presentations (between 2 and 13), the direction of one of the stimuli changed. The monkeys were rewarded for making a saccade to the direction change in either location. We analyzed neuronal responses to all identical stimulus presentations except the first to minimize the effect of adaptation.

b: Illustration of recording locations. Populations of MT and SC neurons were recorded with linear 24-channel moveable probes from the right hemisphere of two monkeys as they were doing the behavioral task described in (a).

c: Receptive field locations of recorded units from an example recording session. The dots represent the receptive field centers of $28 M T$ (red) and $26 S C$ (blue) units. The circles represent the size and location of the median receptive field from each area. 
$\boldsymbol{d}:$ Schematics describing the hypotheses about attention-related changes in information flow between two areas. Each icon depicts the response space of the source area (the responses of the first $n$ neurons or principal components, for instance), and orange and blue surfaces that represent two subspaces for the private or shared fluctuations in neural activity respectively. The two rows of icons represent the attended and unattended conditions (when attention was directed toward or away from the receptive fields of the recorded neurons), and each column describes the expected result of each of the following hypothesis. (left) Attention could alter the dimensionality of the private, shared, or both subspaces. If attention only modified local representations, then the number of private dimensions that explain the local neural fluctuations would change. (middle) Alternatively, attention could modulate information flow by enhancing or diminishing the extent to which neural activity in a target population tracks the neural activity of its source. If attention acted via this mechanism locally, then prediction would improve in private dimensions. (right) If attention modulated functional communication by modulating information flow across areas, then prediction would improve in shared dimensions.

\section{Prediction of SC activity from MT activity using linear models improves with attention}

143 Testing the predictions of our hypotheses requires calculating the ability to predict the activity of

144 one population of neurons from another and identifying the dimensions of neural population

145 space through which functional communication occurs. We plot the results of these analyses for

146 one representative session in Figure 2. We used ridge regression to impose a sparse mapping

147 between random subsets of MT neurons and the full populations of SC neurons in each attention

148 condition (see Methods and Semedo et al, 2019).

150 Several features of this example recording session were typical of our data set. First, no subset of 151 the recorded MT neurons could effectively predict SC neural activity; the prediction accuracy

152 monotonically increased with the addition of MT neurons. Second, the accuracy of prediction 153 was significantly improved in the attend in trials vs attend out trials across all sub-selections of 154 the MT population. Third, attention also improved the ability to predict random subsets of SC 155 neurons from the full population of recorded MT neurons (Figure 2b).

157 To determine the relationship between these measures of functional communication between

158 neuronal populations in MT and the SC and more well-studied effects of attention, we next 159 calculated traditional metrics neuronal activity like pairwise spike count correlations (Figure 2c) 160 and population firing rate (Figure 2d). For this session, attention significantly decreased spike 
161 count correlations in both MT and SC but did not have an effect on variability shared between

162 pairs of neurons in different brain areas. Attention also significantly increased mean firing rates

163 in this session. Firing rate and correlation changes across sessions are detailed in Figure S1.

164

165 For the example session, we observed no attention-related change in the population

166 dimensionality in MT ( 5 dimensions; Figure 2e) and SC ( 3.5 dimensions; Figure 2g) defined

167 as the smallest number of dimensions that captured $95 \%$ of the variance in the shared covariance

168 matrix (assessed using factor analysis; (Cunningham and Yu, 2014); also see Methods for code

169 and other resources).

170

171 We next tested whether, as between two areas of visual cortex (Semedo et al., 2019), interactions

172 between MT and the SC are limited to a subset of dimensions of neural population space. For the

173 example session in Figure 2, only 2-3 dimensions of MT activity (identified using reduced rank

174 regression; see Methods; defined at the number of dimensions at which the curves in Figure $2 \mathrm{f}$

175 reach asymptote) predicted SC activity at least as well as a full linear model (fit using ridge

176 regression; see Methods). The prediction accuracy for the attend in trials was significantly better

177 than the attend out trials irrespective of the number of predictive dimensions (the black line is

178 always above the gray line in Figure 2f). 


\section{Figure 2:}

(full page width -2 column)

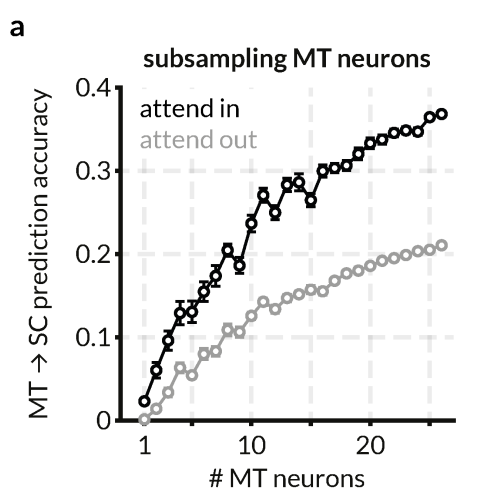

b
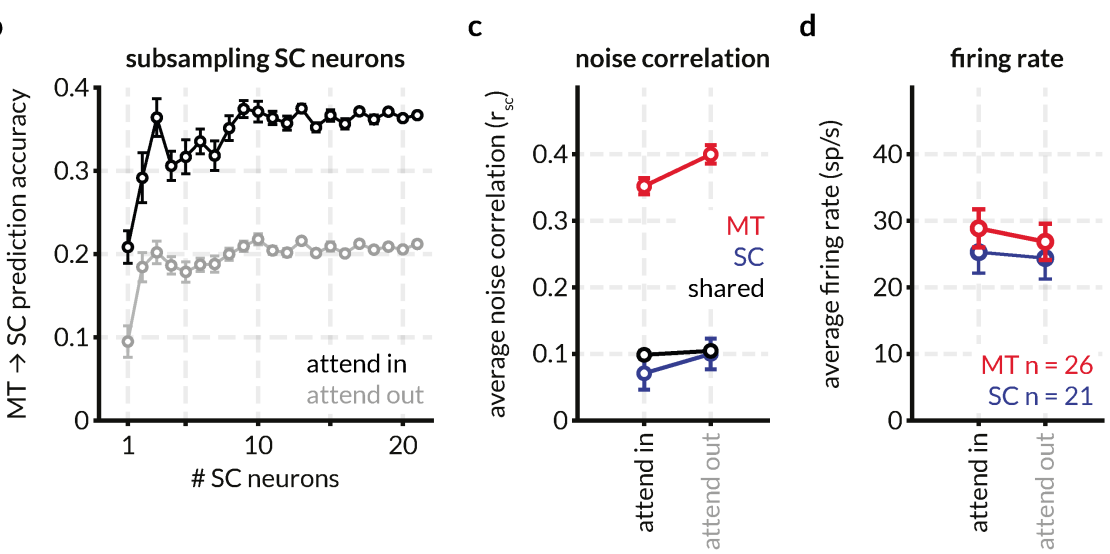

e
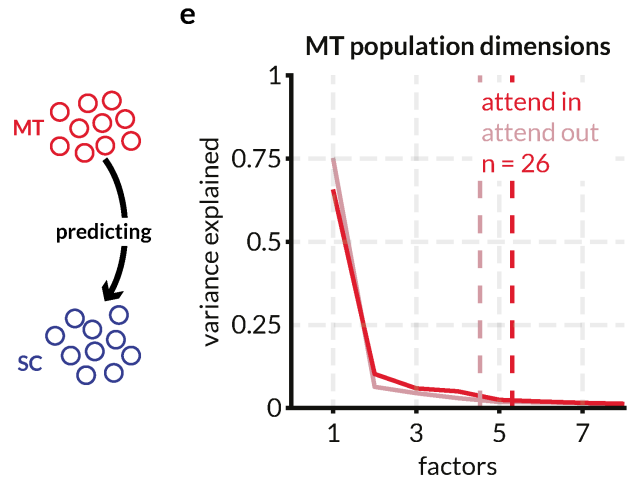

f

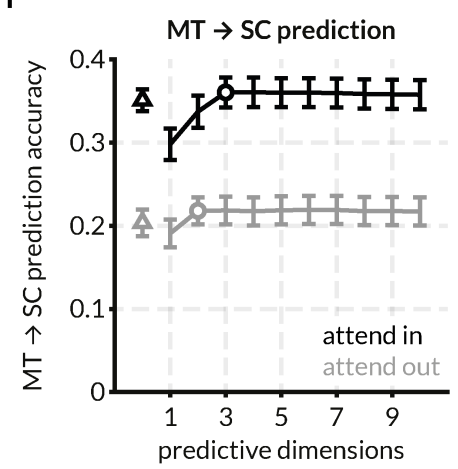

g

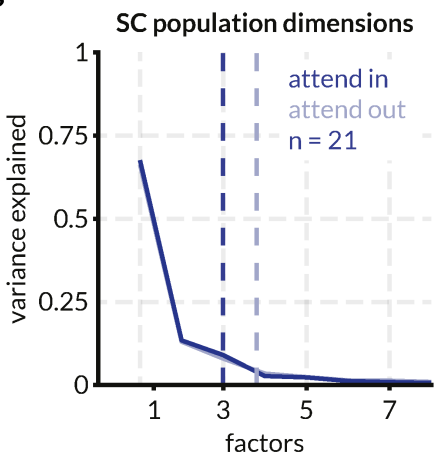

\section{Attention improves prediction of SC activity from MT activity, increases firing rate, and} decreases spike-count correlations in an example recording session.

a: For an example session, the prediction accuracy of 1-26 randomly sampled (without replacement) MT neurons predicting the activity of a population of 21 SC neurons in the two attention conditions (attend in refers to the trials in which attention was directed within the joint RFs of the MT and SC neurons, and attend out refers to trials in which attention was directed in the opposite hemifield). Prediction was performed using a linear model with ridge regression and prediction performance was defined as the average cross-validated normalized square error (NSE) for the smallest ridge parameter for which the performance was within 1 SEM of the peak performance. Each point represents the mean prediction performance for $n$ MT neurons predicting the full population of SC neurons. Error bars represent the standard error of the mean across random subsamples of n neurons.

b: Same as (a) but for predicting random subsets of SC neurons using the activity of the full population of MT neurons, showing that the effect of attention on MT-SC communication is not limited to a subpopulation of the either the MT or SC neurons recorded in this session. $c:$ Spike count correlation $\left(r_{S C}\right)$ defined as the correlation between the responses of pairs of neurons to all stimulus presentations for all MT neurons (325 pairs, red), SC neurons (210 pairs, blue), and MT-SC pairs (546 pairs, black). Attention decreases spike count correlations in MT ( $p$ 
$=1.2 \times 10^{-10}$; Wilcoxon signed rank test $($ WSRT $\left.)\right)$ and SC $(p=0.0206 ;$ WSRT) but has no effect on pairwise correlations across areas $(p=0.2$; WSRT) for this recording session. See Figure S1 for $r_{S C}$ for all pairs across recording sessions.

$\boldsymbol{d}$ : Neuronal firing rates increase with attention in MT $\left(p=8.3 \times 10^{-6} ;\right.$ WSRT $)$ and SC $(p=0.04$; WSRT) for this session. See Figure S1 for firing rates for all neurons across sessions.

$\boldsymbol{e}:$ Factor analysis of MT population responses for this session reveals that $90 \%$ of the variance in the MT response fluctuations can be accounted for by 5 dimensions. The number of population dimensions is greater for the attend in condition vs the attend out condition. The arrow in the icon signifies that the MT population (source) is being used to predict the SC population (target): henceforth labeled as $M T \rightarrow S C$ prediction.

$f$ : Predicting SC activity from MT responses using reduced-rank regression (RRR; black and gray lines) and ridge regression (triangle) reveals that the prediction performance for a matched number of trials is dramatically better for the attend in condition (black) vs the attend out condition (gray). The optimum number of dimensions (circle) for the reduced-rank regression was defined as the lowest number of dimensions for which prediction performance was within 1 SEM of peak performance. This performance is at least as good as the performance of the ridge regression performance that uses all the source dimensions for prediction (the difference between the RRR prediction and the ridge regression prediction was not significant across sessions; data not shown). The number of source dimensions required for optimum regression performance was 3 for attend in and 2 for attend out suggesting that fewer dimensions are required for communication between MT and SC than the total number of population dimensions.

g: Factor analysis of SC neurons reveals that $90 \%$ of the variance in the SC response fluctuations can be accounted for by 3-4 dimensions. For this session, the number of population dimensions is greater for the attend out condition vs the attend in condition.

180 Testing our hypothesized mechanisms of information flow (Figure 1) requires determining how

181 attention affects the dimensionality and informativeness of interactions within and between

182 populations of neurons in MT and the SC. We therefore fit linear models for repeated random

183 splits of the populations of recorded MT and SC neurons in all four directions - MT $\rightarrow$ SC, MT

$\rightarrow \mathrm{MT}, \mathrm{SC} \rightarrow \mathrm{MT}$, and SC $\rightarrow \mathrm{SC}$. We depict the effect of attention on these four communication

185 channels (for the same single session as in Figure 2) in the form of mean prediction accuracy

186 across all tested population splits (Figure 3c-f). For this session, the prediction performance

187 improves with attention for all functional communication channels except within MT where it

188 depreciates. We estimated the population dimensionality as of each of the randomly split 
189 populations of MT and SC neurons using factor analysis to compare with number of predictive

190 dimensions (Figures 3a, b). Consistent with result for the full population above, the number of

191 dimensions within each area is not affected by attention.

\section{Figure 3:}

(full page width -2 column)

a
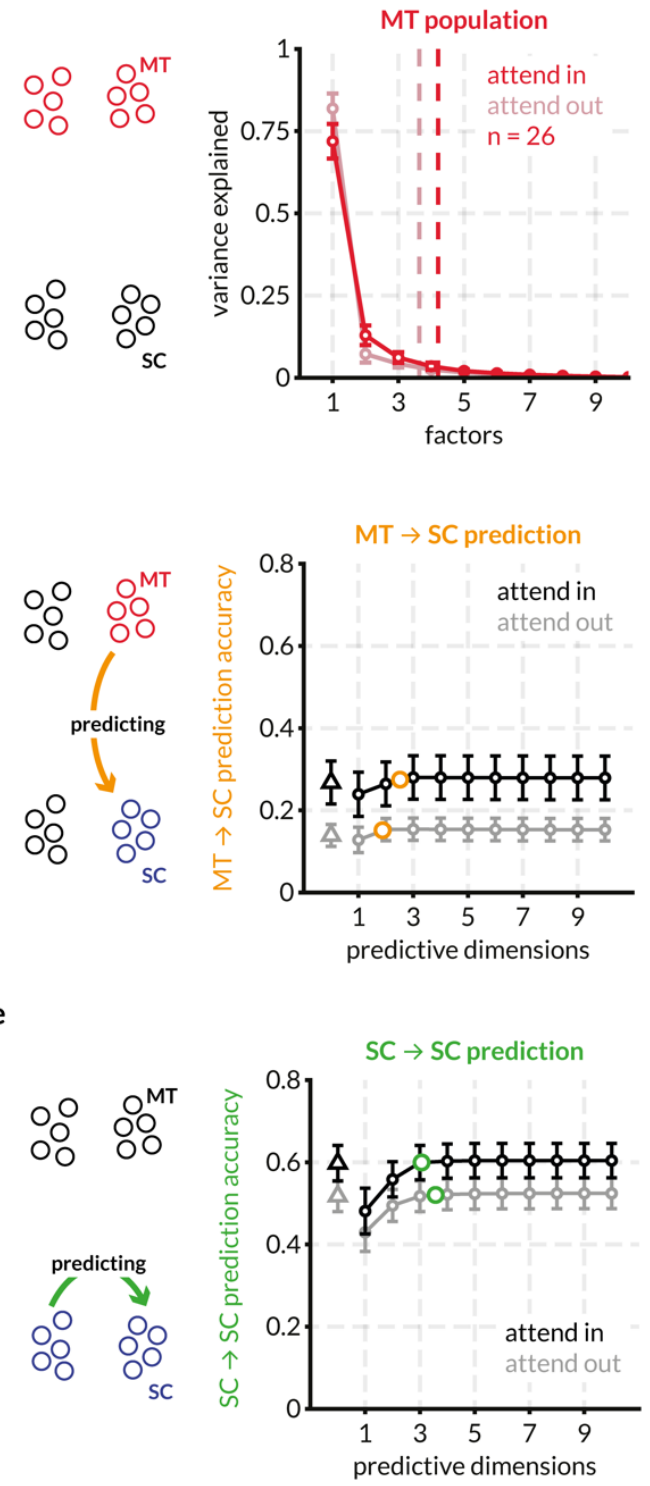

b

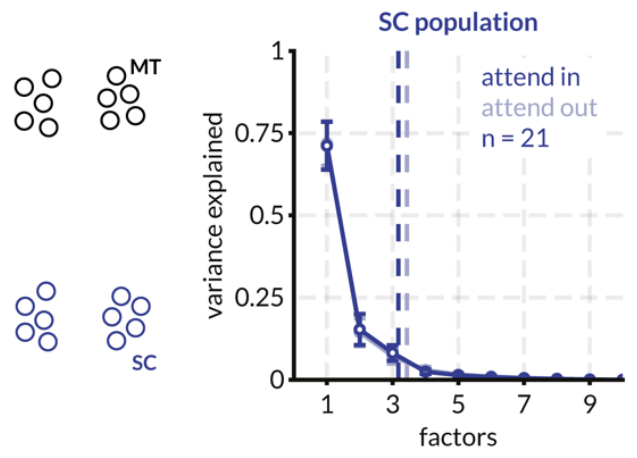

d

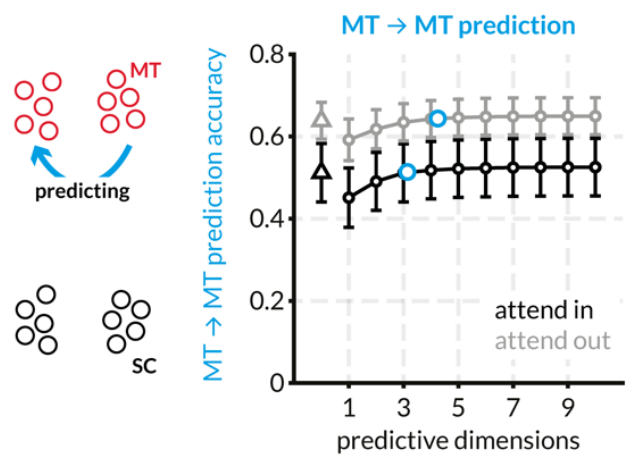

f

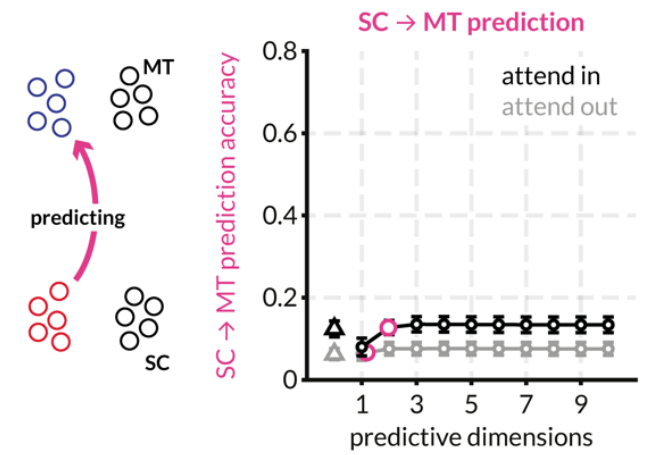

Randomly partitioned populations of MT and SC neurons predict activity within and across areas better with attention for the same example session. To compare prediction performance for inter-and intra-areal interactions, we randomly split both the populations of MT and SC neurons into two halves each - the target and source halves - as indicated in the icons. Each 
source half was used to predict the activity of both target halves using both the full linear model (ridge regression) and the reduced-rank regression (RRR) model. This split was done 20 times and the mean performance across the random splits is shown in c-f. Error bars indicate the SEM across these splits.

a: Factor analysis of MT neurons reveals that $95 \%$ of the variance in the MT response fluctuations can be accounted for by $\sim 4$ dimensions on average across all splits for this session. The number of population dimensions is greater for the attend in condition vs the attend out condition.

b: Same as (a) for SC neurons. For this session, SC population fluctuations are captured by $\sim 3$ dimensions in both attention conditions.

c: Average prediction performance for the full model (black and gray triangles) and the RRR model (black and grey circles) across random splits of the MT and SC populations. The orange circle indicates the average optimum performance and average number of optimum prediction dimensions across the random splits. For each session, this point of optimum performance is plotted in different comparisons in the following figures. For this session, attention improves MT $\rightarrow$ SC prediction performance. For all predictions, the RRR model performs at least on par with the full linear model using ridge regression.

$d:$ same as (c) for $M T \rightarrow M T$ predictions. For this session, attention degrades prediction performance. The average optimum performance and average optimum prediction dimensions are indicated with blue circles.

$\boldsymbol{e}:$ same as (c) for $S C \rightarrow S C$ predictions. For this session, attention improves prediction performance. The average optimum performance and average optimum prediction dimensions are indicated with green circles.

$f:$ same as (c) for $S C \rightarrow M T$ predictions. For this session, attention improves prediction performance. The average optimum performance and average optimum prediction dimensions are indicated with pink circles.

192 Across sessions, prediction performance between MT and the SC improves with attention

193 without changing the dimensionality of that communication (Figure 4). The number of predictive

194 dimensions required to account for intra-areal communications was higher than the number of

195 dimensions for inter areal communication in both attention conditions. Whereas prediction

196 accuracy for intra-areal communication was consistently high and remained unaffected by

197 attention, the prediction accuracy for inter-areal communication significantly improved with

198 attention (Figure 4b, which shows the ratios of the number of predictive dimensions and of the

199 prediction accuracy in the two attention conditions). Attention does not affect the number of

200 predictive dimensions required for communication within and across areas (the marginal

201 distributions of ratios are centered at and not significantly different from 1; Wilcoxon signed 
rank test) but improves the prediction accuracy between MT and the SC (the distributions of ratios of MT $\rightarrow$ MT and SC $\rightarrow$ SC prediction accuracy are centered at and not significantly

204 different from 1 but the ratios of MT $\rightarrow$ SC and SC $\rightarrow$ MT prediction accuracy are significantly

205 greater than 1; Wilcoxon signed rank test; see also the distributions for each communication 206 channel in Figure S3).

\section{Figure 4:}

(0.5-page width -1 column) a

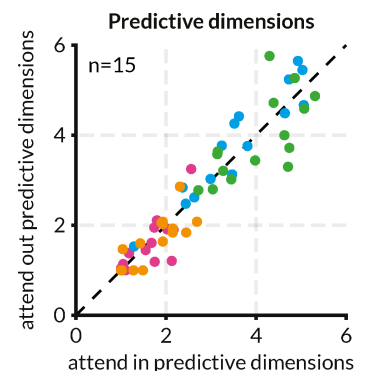

C
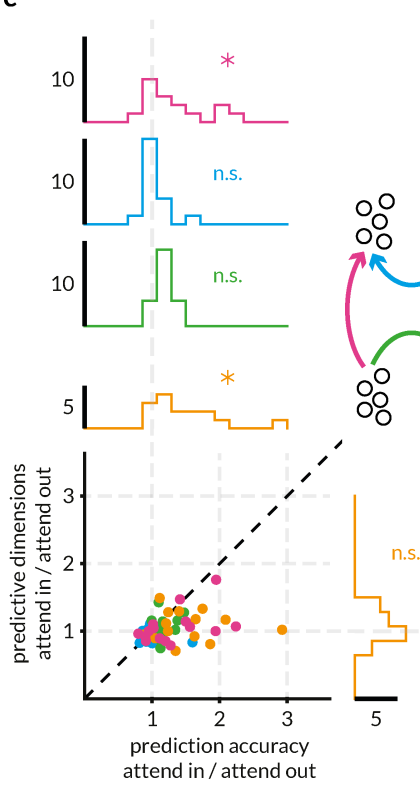

b
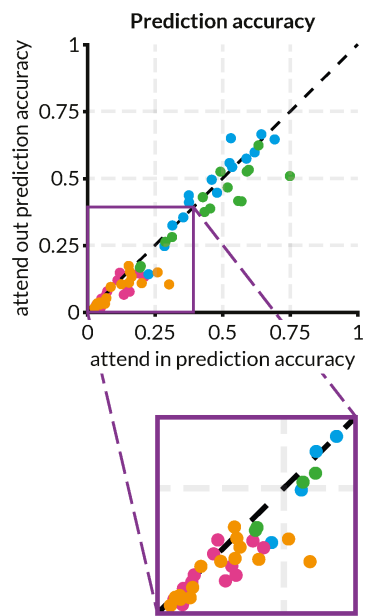
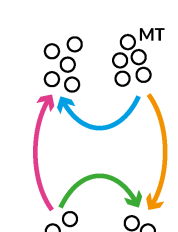

sc

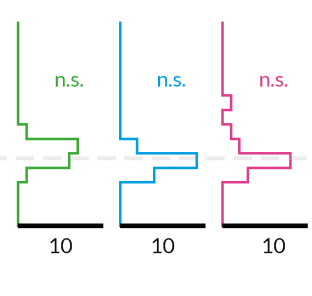

Attention improves the accuracy of across area prediction but not within area prediction without altering the dimensionality of the communication subspace. Each point of a given color represents a recording session. The color scheme is depicted in the icon in (c) and is consistent with other figures.

a: Attention does not affect the dimensionality of the interaction between MT and SC neurons. Each point represents the average number of optimum predictive dimensions for each session for one of the four predictions $M T \rightarrow S C$ (orange), $M T \rightarrow M T$ (blue), $S C \rightarrow$ $S C$ (green), $S C \rightarrow M T$ (pink)-for the two attention conditions. There was so significant difference between the number of predictive dimensions for any of the four predictions. See Figure S5 for a detailed version of this panel. (MT-MT mean 3.67, range 1.5-5.2 for attend in and mean 3.74, range 1.1-5.3 for attend out; SC-SC mean 4, range 2.9-5.3 for attend in and mean 3.9, range 2.85-5.7 for attend out; MT-SC mean 1.8, range 1-2.5 for attend

in and mean 1.75, range 1-2.7 for attend out; SC-MT mean 1.6, range 1-2.7 for attend in and mean 1.55, range 1-3.15 for attend out)

b: Attention significantly increases the prediction accuracy of inter-areal but not intra-areal interactions. Each point represents the average prediction performance across random splits for one of the four predictions. The purple inset affords a zoomed in view of the relevant part of the plot which reveals that the points corresponding to the MT $\rightarrow S C$ (orange) and SC $\rightarrow M T$ (pink) 
predictions lie below the unity line. The average prediction accuracies for the attend in trials were significantly greater than those for the attend out trials for the MT $\rightarrow$ SC prediction ( $p=$ 0.0015; Wilcoxon signed-rank test) and for the $S C \rightarrow M T$ prediction $\left(p=8.54 \times 10^{-4}\right.$; Wilcoxon signed-rank test) but not the MT $\rightarrow$ MT or SC $\rightarrow$ SC predictions.

$c:$ The data in $(a)$ and $(b)$ visualized as a ratio of attend in and attend out. The marginal distributions of the ratios of prediction accuracy and predictive dimensions for all four predictions are also displayed. The mean ratios of prediction accuracy for $M T \rightarrow S C$ (orange) and $S C \rightarrow M T$ (pink) were significantly greater than 1 ( $p=0.0016$ and $p=0.012$ respectively; $t$ test). The colored arrows in the icon indicate the source and target populations for each of the four predictions.

207 While attention is known to affect the mean pairwise spike count correlations within and 208 between areas (Cohen and Maunsell, 2009; Mitchell et al., 2009; Ruff and Cohen, 2014a, 2016a), 209 we found that attention-related improvements in prediction accuracy are not contingent on 210 increases or decreases in spike count correlations. The ratio of prediction accuracies in the two 211 attention conditions within and between areas was unrelated to the attention-related difference in 212 mean spike count correlations between pairs of neurons within MT, within SC and between MT 213 and SC (Figure S3).

215 The connectivity and functional roles of populations of SC neurons differ by layer, so we made 216 use of our recordings that spanned layers to investigate whether functional communication 217 between MT and the SC depends on layer as well. MT projections to SC predominantly end in 218 the superficial layers in SC ((Fries, 1984, 1985) but also see (Lock et al., 2003)). Tecto-pulvinar 219 projections from the superficial and intermediate layers of SC end in the inferior pulvinar which 220 in turn projects to extra-striate areas (Lyon et al., 2010; Stepniewska et al., 1999). Also, there is 221 some evidence that extra-striate projecting lateral geniculate nucleus (LGN) neurons do not 222 receive direct retinal input and are dependent on SC projections across all layers for relaying 223 visual information to MT (Benevento and Yoshida, 1981; Rodman et al., 1990). Given these 224 laminar differences in cortical and thalamic inputs to and outputs from SC, we tested whether 225 there is a difference between the attentional effect on information flow across functional classes 226 of SC neurons. To classify SC neurons, we calculated an oculo-motor score based on SC neuron 227 responses to visual stimuli and responses just prior to saccade onset (see Methods) and divided 228 each population into two groups based on the rank ordering of oculo-motor scores. We then 
further split each SC population randomly as described before to serve as the source and target of

230 regression with the simultaneously recorded MT population (Figure S4). We found no significant

231 differences in the effect of attention on either the prediction accuracy or the number of

232 dimensions required for prediction between the SC populations split by oculo-motor score

233 (labeled visual and motor for brevity). Compared to random splits of the SC population, when

234 split by oculo-motor score, the effect of attention on the prediction accuracy of the SC $\rightarrow$ SC

235 regression is pronounced (Figure S3c vs Figure S4c).

\section{Attention does not improve information flow by altering private or communication}

238 subspaces

239 The attention-related improvement in information flow (as implied by increased prediction

240 accuracy across MT and SC) could in principle arise by changing the subspaces of activity

241 responsible for functional communication within or between areas. We did not find evidence that

242 attention changes the dimensionality of any of these subspaces: there was no attention-related

243 change in the dimensionality of the local populations of MT and SC neurons (Figure S5a and

244 S5b respectively) or in the number of predictive dimensions for the various communication

245 subspaces within and between the two areas (Figure S5c-f). We consistently found that more

246 dimensions were required to account for intra-areal communication than to account for inter-

247 areal communication (mean 3.6 for MT $\rightarrow$ MT and 4 for SC $\rightarrow$ SC; vs 1.8 for MT $\rightarrow$ SC and 1.6

248 for SC $\rightarrow$ MT). This disparity suggests that MT and SC interact via a limited communication

249 subspace.

251 Attention did not affect the dimensionality of the communication subspace. When we compared

252 the number of dimensions used for private communication with the number of shared dimensions

253 for MT $\rightarrow$ MT prediction and MT $\rightarrow$ SC prediction, we found that significantly fewer

254 dimensions are required for MT $\rightarrow$ SC communication than are available, but MT $\rightarrow$ MT

255 communication utilizes all available dimensions (Figure 5a). This effect was similar in the two

256 attention conditions (Figures $5 \mathrm{~b}$ and c), and we found similar results in the SC $\rightarrow$ MT direction

257 when compared with SC $\rightarrow$ SC communication (Figure 5d-f). We found no relationship between

258 the functional communication channels when assessed on a session-by-session basis (Figure S6). 
We also did cross-prediction analyses (using the attend in linear model to predict attend out data and vice versa) to check if the structure of the communication subspace changes while keeping

261 its dimensionality, in turn causing the prediction accuracy to be better (Figure S7). We found that

262 while the intra-areal models performed almost as well when swapped, the inter-areal models

263 suffered a loss in prediction accuracy. This does not necessarily imply that the geometry of the

264 communication subspaces changes with attention but that linear methods are unable to find a

265 common subspace between the two attention conditions (also see Discussion).

\section{Figure 5:}

(full page width -2 column)

a

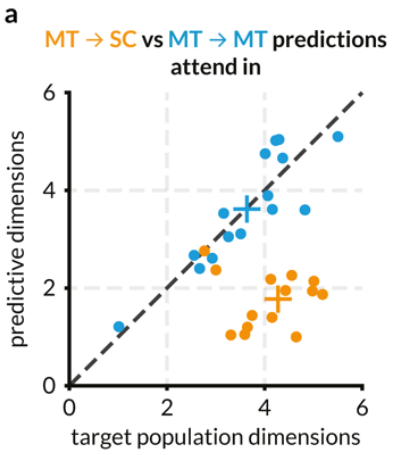

b

$\mathrm{MT} \rightarrow \mathrm{SC}$ vs $\mathrm{MT} \rightarrow \mathrm{MT}$ predictions

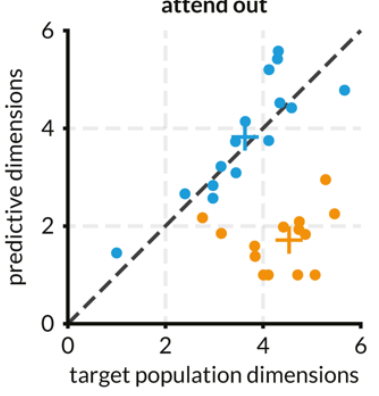

c

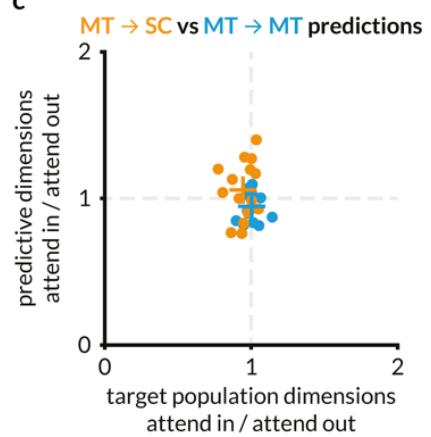

d $\mathrm{SC} \rightarrow \mathrm{MT}$ vs SC $\rightarrow$ SC predictions attend in

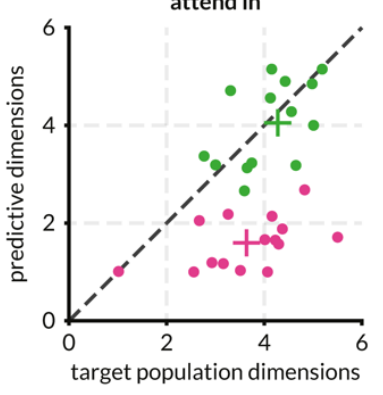

$\mathrm{SC} \rightarrow \mathrm{MT}$ vs SC $\rightarrow$ SC predictions

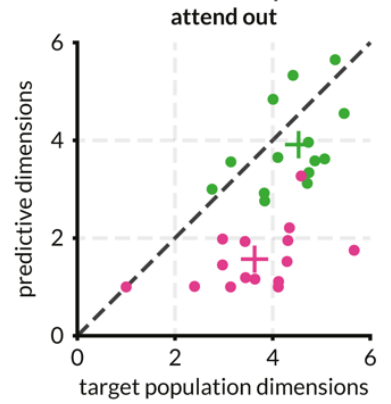

f

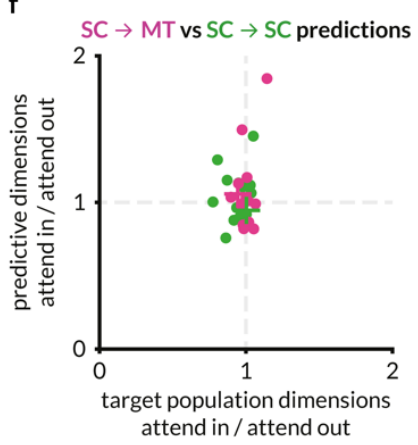

$M T$ and SC populations interact via a communication subspace, but attention has no effect on the dimensionality of the communication subspace. Each point represents a recording session, and the color scheme is the same as other figures. Colored + represents the mean of the corresponding points. This figure compares the number of factors that explain $95 \%$ of the variance in the target area (from factor analysis) with the number of dimensions in the source area that are sufficient to predict the target area activity (from RR regression). Qualitative comparisons between the absolute values of the 'number of dimensions' from these two analyses in depicted in $a, b, d$, and $e$. The effect of attention is depicted in $c$ and $f$. 
a: For the attend in condition, the number of private predictive dimensions are greater than the number of shared predictive dimensions in MT. Further, for the MT $\rightarrow$ SC prediction (orange points), fewer dimensions are required to predict SC activity than are required to explain $95 \%$ of the variance in the SC activity, forming a communication subspace in MT that comprises of $\sim 2$ shared dimensions that are sufficient to predict the $\sim 4$-dimensional activity in SC.For the MT $\rightarrow M T$ prediction, the number of predictive dimensions is similar to the number of population dimensions i.e., the predictive dimensions in MT are as large as possible and closely match the complexity of the target population, unlike the $M T \rightarrow S C$ prediction.

b: Same as (a) for the attend out condition.

c: Data in (a) and (b) presented as a ratio to compare the effect of attention on the communication subspace in $M T$.

$d:$ For the attend in condition, the number of private predictive dimensions are greater than the number of shared predictive dimensions in SC. For the $S C \rightarrow M T$ prediction (pink points), fewer dimensions are required to predict MT activity than are required to explain $95 \%$ of the variance in the MT activity i.e., a communication subspace exists in $S C$ that comprises of $\sim 2$ shared dimensions that are sufficient to predict the $~ 3.5$-dimensional activity in MT.

$\boldsymbol{e}:$ Same as $(d)$ for the attend out condition.

$f:$ Data in $(d)$ and (e) presented as a ratio to compare the effect of attention on the communication subspace in SC.

\section{Attention improves information flow between V1 and MT}

267 Both MT and SC exhibit relatively large attention-related changes in a number of measures of 268 neuronal activity (Goldberg and Wurtz, 1972; Ignashchenkova et al., 2004; Krauzlis et al., 2013;

269 Recanzone and Wurtz, 2000; Seidemann and Newsome, 1999; Womelsdorf et al., 2006b).

270 Attention-related improvements in information flow may in principle be exclusive to pairs of

271 regions that individually show significant changes in local representations. We tested this

272 hypothesis by analyzing previously published simultaneous recordings of populations of neurons

273 in V1 (which tend to show very modest effects of attention) and a single MT neuron (Ruff and

274 Cohen, 2016a, 2016b; Ruff et al., 2016). As with the MT $\rightarrow$ SC results, we found that attention

275 dramatically improves V1 $\rightarrow$ MT prediction accuracy (Figure 6c; because we only recorded one

276 MT neuron at a time, it was not possible to compute MT $\rightarrow$ V1 prediction accuracy). These

277 results demonstrate that even though the effect of attention on V1 was small, attention-related

278 effects on inter-areal communication are not contingent on large effects of attention in individual 279 regions. 


\section{Figure 6:}

(full page width -2 column)
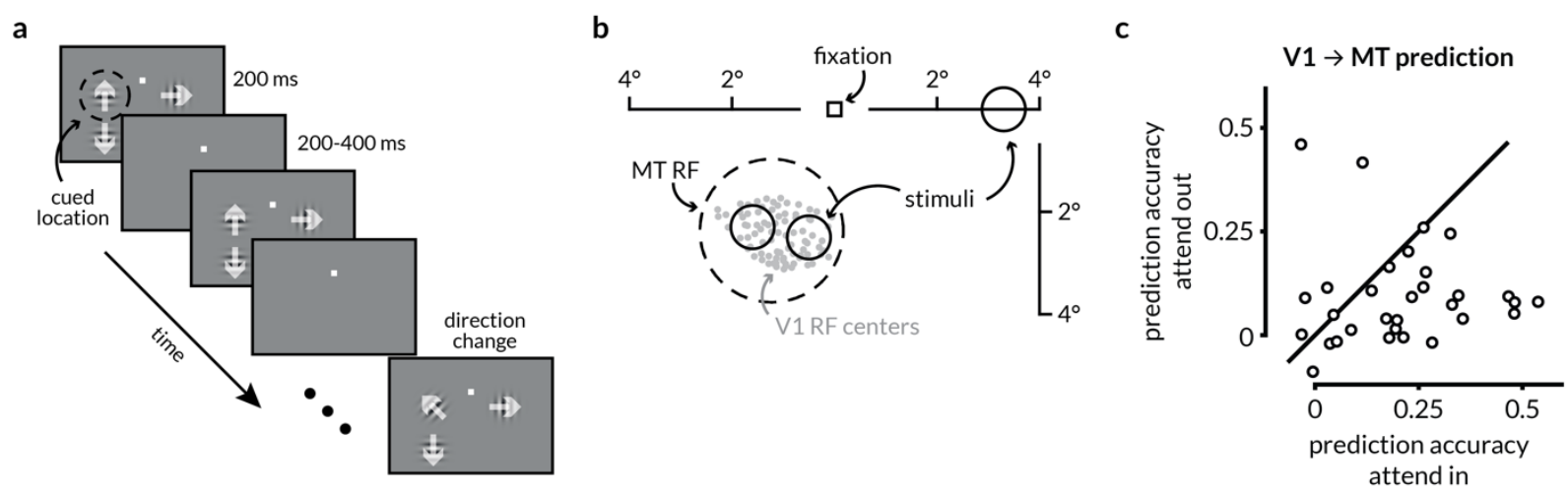

\section{Attention enhances prediction accuracy between V1 and MT.}

a: Schematic of the motion direction change detection task used during the V1-MT recordings. The monkeys were instructed to attend to changes in motion direction at one of three spatial locations while ignoring changes at the other two locations in blocks of 50-100 trials. The monkey started the trial by fixating a central spot. Two or three small Gabor stimuli synchronously flashed on for 200ms and off for a randomized 200-400ms period. Two of the stimuli were positioned inside the joint receptive fields (RFs) of the V1 and MT neurons, and the other was in the opposite hemifield. Trials during which attention was directed into the MT RF towards either of the two spatial locations were considered attend in trials, and trials in which attention was directed to the opposite hemifield were considered attend out trials. In blocks when the monkey was cued to attend to one of the two locations inside the RFs, the third stimulus wasn't presented. One of the two stimuli in the RF moved in the preferred direction of recorded MT neuron and the other moved in the anti-preferred direction. When presented, the third stimulus moved in the preferred direction of the MT neuron. After a randomized number of stimulus presentations (between 2 and 13), the direction of one of the stimuli changed. The monkeys were rewarded for making a saccade to the direction change in the cued location. Premature saccades or saccades to changes in motion direction at the un-cued location were not rewarded. We analyzed all identical stimulus presentations except the first to minimize the effect of adaptation.

b: RF locations of recorded units from an example recording session. The gray dots represent the RF centers of $96 \mathrm{VI}$ neurons. The dotted circle represents the size and location of the RF for the recorded MT neuron. The size and locations of the stimuli were selected such that they lie within the MT RF.

$c:$ Attention improves the performance of VI $\rightarrow$ MT prediction. Each dot represents the crossvalidated normalized $r^{2}$ for a linear model of the MT neuron's activity from V1 population activity using ridge regression for one recording session. The prediction accuracy on attend in trials was significantly greater than the accuracy on attend out trials ( $p=0.0159$; Wilcoxon 
signed-rank test). The value of the ridge parameter was chosen to be the smallest value for which the model performance was within 1 S.E.M. of the peak performance.

\section{Discussion}

281 Our results show that attention changes the functional communication between populations of

282 visual and premotor neurons. We demonstrated that attention changes the extent to which the 283 activity of populations of neurons in the SC and be predicted by neuronal population in MT, and

284 vice versa. These changes in information flow are not accompanied by changes in the

285 dimensionality of the subspace of activity that is shared between areas, and they are independent

286 of changes in firing rates, noise correlations, or population activity within each brain area. These

287 results suggest that changes in information flow may mediate behavioral flexibility and place

288 important constraints on models of flexible neural circuits.

How attention-related increases in functional communication fit in with hypothesized mechanisms underlying attention

292 Previous studies have focused on a small number of hypothesized mechanisms by which

293 attention might improve perception (Driver, 2001; Lavie, 2010; Peelen and Kastner, 2014; Ruff

294 et al., 2018). The most studied hypothesis is that attention improves perception by improving

295 information encoding (Cohen and Maunsell, 2009; Mitchell et al., 2007, 2009; Ruff and Cohen,

296 2014a). The observed attention-related changes in the responses of individual neurons and in

297 correlations between visual neurons appear consistent with this hypothesis. However, neuronal

298 populations typically encode more than enough sensory information to account for

299 psychophysical performance (Kanitscheider et al., 2015; Kohn et al., 2016b; Parker and

300 Newsome, 1998; Ruff and Cohen, 2014b, 2019), and the changes in trial-by-trial fluctuations

301 may not reflect changes in information coding that are behaviorally-relevant (Baruni et al., 2015;

302 Moreno-Bote et al., 2014). An alternate theory is that attention selectively improves the

303 communication of sensory information to the neurons involved in perceptual decision-making.

304 Physiological studies along these lines have primarily focused on changes in synchrony or

305 coherence between areas on very short timescales (one or a few milliseconds, for review see

306 (Womelsdorf and Fries, 2007)) or using human imaging data to assess functional connectivity

307 over multiple seconds (Indovina and Macaluso, 2004; Ozaki, 2011; Rossi et al., 2014). However, 
co-variability on short timescales is mathematically nearly independent of correlations on the timescale of hundreds of milliseconds (Bair et al., 2001), and unlike fluctuations on very short or very long timescales, response fluctuations on the timescale of hundreds of milliseconds covary with perceptual decisions (Nienborg and Cumming, 2010; Nienborg et al., 2012).

313 Recently, we showed that attention is associated with only modest changes in either information

314 coding in visual cortex or the way information is read out by premotor neurons on the timescale

315 of perceptual decisions (Ruff and Cohen, 2019). Instead, our multi-neuron, multi-area recordings

316 suggest that attention reshapes population activity in visual cortex which changes the visual

317 information that guides behavior via relatively fixed readout mechanisms. Our current results

318 suggest a functional implication of this reshaping, changing the information that is shared

319 between sensory neurons and the premotor neurons involved in decision-making, without

320 substantially changing the geometry of the subspace of activity that is shared between them.

\section{The communication subspace as a mechanism for flexible behavior}

323 Many recent studies have shown that the activity of populations of neurons in many areas is

324 generally confined to a subspace of population activity that is much lower dimensional than the

325 number of recorded neurons (Cowley et al., 2016; Cunningham and Yu, 2014; Elsayed and

326 Cunningham, 2017; Elsayed et al., 2016; Golub et al., 2016; Jazayeri and Afraz, 2017; Kaufman

327 et al., 2014; Kiani et al., 2007; Miri et al., 2017; Morcos and Harvey, 2016; Pandarinath et al.,

328 2018; Pitkow and Angelaki, 2017; Ruff et al., 2018; Sadtler et al., 2014; Yu et al., 2009). The

329 divergent anatomical connections between even the most highly interconnected brain areas have

330 long suggested that only a portion of the information encoded in each area is shared between

331 areas.

333 A recent set of studies used recordings from multiple populations of neurons to establish that

334 functional communication between different brain areas in the motor (Kaufman et al., 2014) or

335 visual system (Semedo et al., 2019, 2021) is confined to a subspace of activity that is even lower

336 dimensional than the activity within each area. Our results are consistent with the proposal in

337 these that this limited communication subspace is an attractive mechanism for behavioral

338 flexibility (Kaufman et al., 2014; Semedo et al., 2019). Because only a subset of information is 
shared, reshaping activity within the source area (as in Ruff and Cohen, 2019) and/or having a

340 fixed but nonlinear subspace (proposed in Semedo et al., 2019) would change the information

341 that is functionally communicated to a target area. Using cross-prediction analyses, we found

342 that these linear methods reveal a difference in the structure of the communication subspace

343 across attention conditions, but this observation may be consistent with a fixed, non-linear

344 communication subspace, information flow could be improved by shifting the alignment of the

345 shared fluctuations along the non-linearity (Figure S7). This mechanism is particularly attractive

346 because changes in functional communication could occur without relying on changes in the

347 weights relating one population to another, which may rely on synaptic plasticity mechanisms

348 that occur over longer than behaviorally relevant timescales (Egeth and Yantis, 1997).

350 Our results demonstrate that the amount of information shared via the communication subspace

351 between visual areas (V1 and MT, Figure 6) or between visual and premotor areas (MT and the

352 SC, Figure 4) is in fact flexible. In future studies, it will be interesting to test the limits of this

353 flexibility, such as whether this mechanism might mediate flexible communication of different

354 stimulus features or information accumulated on different timescales that must mediate more

355 complex forms of behavioral flexibility.

\section{Constraints on mechanistic models}

358 Measurements of the activity of large populations has proven critical for constraining

359 mechanistic models. Phenomenological models can explain attention-related changes in firing

360 rates (Boynton, 2009; Ecker et al., 2016; Gilbert and Sigman, 2007; Maunsell, 2015;

361 Navalpakkam and Itti, 2005; Reynolds and Heeger, 2009), but these do not provide insight into

362 circuit mechanisms. A staggering variety of biophysical models can recreate the effects of

363 attention on the trial-averaged responses of individual neurons (Ardid et al., 2007; Buia and

364 Tiesinga, 2008; Deco and Thiele, 2011; Huang et al., 2019; Kanashiro et al., 2017; Silver, 2010;

365 Sutherland et al., 2017). We and others have shown that attention-related changes in correlated

366 variability that resides in a low dimensional subspace of population activity provides much

367 stronger constraints on circuit models (Huang et al., 2019; Kanashiro et al., 2017). 
369 The observations that functional communication between areas is lower dimensional than

370 activity within each area (Kaufman et al., 2014; Semedo et al., 2020) and our observation that

371 attention changes this communication will further constrain circuit models. In particular, many

372 models (Brunel and Wang, 2001; Huang et al., 2019; Kanashiro et al., 2017; Machens et al.,

373 2005; Rubin et al., 2015) and experiments (Fu et al., 2014; Karnani et al., 2016; Kuchibhotla et

374 al., 2017) implicate inhibition in the flexibility of neuronal populations, but whether these

375 mechanisms readily create low dimensional and flexible communication subspaces is unknown.

376 It is possible that the complementary influence of different subtypes of inhibitory interneurons

377 may underlie the flexible functional communication we observed (Cardin et al., 2009; Herrero et

378 al., 2008; Roberts et al., 2005; Veit et al., 2017).

\section{Concluding remarks}

381 The hallmark of the nervous system is its flexibility. Flexible behavior must rely, on some level, 382 on flexible information flow. Attention, which changes the behavioral importance of different

383 objects, features, or locations, is a good model of flexible information flow. Our results

384 demonstrate that this flexibility is instantiated, at least in part, by changes in the information that

385 is shared between different stages of the visuomotor pathway. These results lay the groundwork

386 for establishing the role of flexible inter-area communications in a variety of sensory, cognitive,

387 and motor computations.

389 Acknowledgements: We are grateful to K. McKracken for providing technical assistance, to 390 Adam Kohn for comments on an earlier version of this manuscript, and to Brent Doiron, Joao

391 Semedo, and Byron Yu for helpful comments and suggestions regarding data analysis. M.R.C. is

392 supported by National Institutes of Health grant R01EY022930 and Simons Foundation Grant 393 542961SPI.

\section{Author Contributions:}

395 Conceptualization, Methodology, Writing - Review \& Editing, R.S., D.A.R., and M.R.C.;

396 Software, R.S., D.A.R.; Analysis, Visualization, Writing - Original Draft, R.S. and M.R.C.;

397 Funding Acquisition, Resources, Supervision, M.R.C.

398 Declaration of Interests: The authors declare no competing interests. 


\section{STAR $\star$ METHODS}

KEY RESOURCES TABLE

RESOURCE AVAILABILITY

Lead Contact

Materials Availability

Data and Code Availability

EXPERIMENTAL MODEL AND SUBJECT DETAILS

METHOD DETAILS

Electrophysiological Recordings and Behavioral Task

\section{QUANTIFICATION AND STATISTICAL ANALYSIS}

Subsampling

Noise correlations

Regression

Cross-condition, cross-validated regression

Factor Analysis 


\begin{tabular}{|c|c|c|}
\hline REAGENT or RESOURCE & SOURCE & IDENTIFIER \\
\hline \multicolumn{3}{|c|}{ Experimental Models: Organisms/Strains } \\
\hline $\begin{array}{l}\text { Rhesus Macaques (Macaca } \\
\text { mulatta) }\end{array}$ & $\begin{array}{l}\text { University of Pittsburgh and } \\
\text { Carnegie Mellon University }\end{array}$ & $\mathrm{N} / \mathrm{A}$ \\
\hline \multicolumn{3}{|l|}{ Software and Algorithms } \\
\hline MATLAB & MathWorks & $\begin{array}{l}\text { mathworks.com/products/matlab.ht } \\
\underline{\mathrm{ml}}\end{array}$ \\
\hline Psychophysics Toolbox v3 & (Brainard, 1997) & psychtoolbox.org \\
\hline \multicolumn{3}{|l|}{ Data Acquisition } \\
\hline $\begin{array}{l}\text { Plexon 24-channel linear } \\
\text { probes }\end{array}$ & $\begin{array}{l}\text { Plexon Inc } \\
\text { Dallas, TX } 75206 \text { USA }\end{array}$ & $\begin{array}{l}\text { plexon.com/products/plexon-s- } \\
\text { probe/ }\end{array}$ \\
\hline Blackrock 10x10 array & $\begin{array}{l}\text { Blackrock Microsystems LLC } \\
\text { Salt Lake City, UT } 84108 \text { USA }\end{array}$ & $\underline{\text { blackrockmicro.com }}$ \\
\hline Ripple Neuromed & $\begin{array}{l}\text { Ripple Neuromed } \\
\text { Salt Lake City, UT } 84106 \text { USA }\end{array}$ & rippleneuromed.com \\
\hline $\begin{array}{l}\text { EyeLink } 1000 \text { Eye tracking } \\
\text { system }\end{array}$ & $\begin{array}{l}\text { SR Research } \\
\text { Ottawa, Ontario, Canada., K2L } \\
\text { 2B9 }\end{array}$ & $\underline{\text { sr-research.com }}$ \\
\hline Plexon Offline Sorter ${ }^{\mathrm{TM}} 3.3 .2$ & $\begin{array}{l}\text { Plexon Inc } \\
\text { Dallas, TX } 75206 \text { USA }\end{array}$ & plexon.com/products/offline-sorter \\
\hline
\end{tabular}

401 Requests for resources should be directed to and will be fulfilled by the Lead Contact,

402 Ramanujan Srinath (ramanujan@ pitt.edu).

403 Materials Availability

404 This study did not generate new unique reagents.

405 Data and Code Availability 
The data and MATLAB code that support the findings of this study have been deposited in a public Github repository https://github.com/ramanujansrinath/mt-sc-comm-data. MATLAB code for reduced-rank regression and factor analysis has been publicly available by Byron Yu and can be downloaded from https://users.ece.cmu.edu/ byronyu/software.shtml. Further information and requests for data or custom MATLAB code should be directed to and will be fulfilled by the Lead Contact, Ramanujan Srinath (ramanujan@pitt.edu).

\section{EXPERIMENTAL MODEL AND SUBJECT DETAILS}

414 The electrophysiological data in this manuscript comes from two previously reported

415 experiments (Ruff and Cohen, 2016a, 2019). In both experiments, two adult male rhesus

416 monkeys (Macaca mulatta, 8 and $9 \mathrm{~kg}$ ) were used. We implanted each animal with a titanium

417 head post before behavioral training. We identified each cortical area by visualizing the sulci

418 during array implantation, using stereotactic coordinates, and by observing the transition of grey

419 and white matter signals on the movable probes. All animal procedures were approved by the

420 Institutional Animal Care and Use Committees of the University of Pittsburgh and Carnegie

421 Mellon University.

METHOD DETAILS

\section{Electrophysiological Recordings and Behavioral Task}

425 Our methods for presenting visual stimuli and monitoring behavior have been described 426 previously. Briefly, we presented visual stimuli using custom software (written in MATLAB 427 using the Psychophysics Toolbox v3 (Brainard, 1997) on a cathode-ray tube monitor (calibrated 428 to linearize intensity; $1,024 \times 768$ pixels; $120 \mathrm{~Hz}$ refresh rate) placed $54 \mathrm{~cm}$ from each animal. 429 We monitored eye position using an infrared eye tracker (EyeLink 1000; SR Research) and 430 recorded eye position and pupil diameter (1,000 samples/s), neuronal responses (30,000 431 samples/s) and the signal from a photodiode to align neuronal responses to stimulus presentation 432 times (30,000 samples/s) using hardware from Ripple. All spike sorting was done offline 433 manually using Offline Sorter (version 3.3.2; Plexon). We based our analyses on both single 434 units and multiunit clusters and use the term unit to refer to either. 
MT-SC recordings: We implanted two recording chambers on the right hemisphere that granted access to MT and SC for recordings with linear 24-channel moveable probes (Plexon; interelectrode spacing in $\mathrm{MT}=50 \mu \mathrm{m}, \mathrm{SC}=100 \mu \mathrm{m}$ ) and simultaneously recorded activity from neurons in MT and SC that had overlapping spatial receptive fields (Figure 1). To account for visual latencies in the two areas, spikes were counted between 50 and 250ms after stimulus onset. We only analyzed a recorded MT unit if its stimulus-driven firing rate was $10 \%$ higher than its firing rate as measured in the $100 \mathrm{~ms}$ before the onset of the first stimulus. We only analyzed a recorded SC unit if its stimulus-driven firing rate was $10 \%$ higher than its firing rate as measured in the $100 \mathrm{~ms}$ before the onset of the first stimulus or if its response during a $100 \mathrm{~ms}$ epoch before a saccade on hit (correct) trials to the contralateral side was $10 \%$ larger than that same baseline. The dataset consisted of a total of 306 responsive MT units (6-29 units per session, mean 20.4) and 345 responsive SC units (12-29 units per session, mean 23) across 15 recording sessions. Each session began with receptive field mapping using a delayed-saccade task, and direction tuning during passive fixation, followed by multiple blocks of the following attention task. Each block began with a set of trials that instructed the monkey to attend to one of two spatial locations on the screen - either within the joint receptive fields of the neurons or in the opposite hemifield. Following that, each trial began when the monkey acquired fixation on a central spot within a $1.25^{\circ}$ fixation window after which two peripheral drifting Gabor stimuli

454 (one overlapping the receptive fields of the recorded neurons, the other in the opposite visual 455 hemifield) synchronously flashed on (for 200ms) and off (for a randomized period between 200 and 400ms) between 3-12 times before, at a random, unsignaled time, the direction of one of the

457 stimuli changed from that of the preceding stimulus. The monkey reported the orientation change 458 by making a saccade to the changed stimulus within $450 \mathrm{~ms}$ and received a juice reward. Each 459 block consisted of approximately 100 completed trials (i.e., trials that ended in a hit or miss) 460 after which the cued location of the orientation change switched to the other hemifield. Stimulus 461 presentations during the response period of which the monkey made a micro-saccade were excluded from analysis. Neural responses to all stimulus presentations after the first (to minimize 463 the effect of adaptation) and before the orientation change were analyzed. For each session, 464 stimulus presentations were sampled such that the number of presentations was equal for each attention condition. Each session yielded 547-1909 (mean 1277) presentations for each attention 
condition. For each session, SC neurons were divided evenly into oculo-motor (visual for brevity) and motor neurons based on an oculo-motor score calculated as

$$
\text { score }_{\text {vis/mot }}=R_{\text {vis }}-R_{\text {mot }} / R_{\text {vis }}+R_{\text {mot }}
$$

where $R_{v i s}$ is the average neural response to the onset of the first stimulus, and $R_{m o t}$ is the average response prior to a saccade to the target in the contralateral hemifield. This score was calculated for the trials where attention was directed into the joint RFs.

V1-MT recordings: We implanted a 10x10 chronic microelectrode array (Blackrock

474 Microsystems) in V1 and a recording chamber to access MT. Each recording session began with 475 searching a well-isolated MT neuron such that its receptive field (RF) overlapped the population 476 RF of the V1 neurons and was driven similarly above baseline by a single stimulus flashed in 477 each of two chosen locations. This dataset consisted of a total of 1631 responsive V1 units and 47832 responsive MT units (1 unit per session in MT, 7-83 units per session, mean 51 in V1) across 47932 recording sessions. Each block of trials began with a set of trials that instructed the monkey to 480 attend to one of three spatial locations on the screen - either one of two locations within the 481 receptive field of the MT neuron or one in the opposite hemifield. Each trial began when the 482 monkey acquired fixation on a $1^{\circ}$ fixation window. For blocks in which attention was directed 483 within the RF of the MT neuron, two achromatic Gabor stimuli of equal contrast, spatial 484 frequency, and speed were presented drifting in opposite directions (preferred and null direction 485 for the MT neuron). For blocks in which attention was directed to the opposite hemifield, a third 486 drifting Gabor was similarly flashed at the cued location. In these blocks, the contrast of the 487 stimulus at the cued location was different from the two stimuli within the RF of the MT neuron. 488 This was done to study the stimulus dependence of spike count correlations across cortical areas 489 but is not critical to the current analyses as here the comparison is between the trials where 490 attention is directed either into or out of the RF of the MT neuron regardless of stimulus 491 parameters or specific location with the RF. After 2-14 presentations of the same stimuli, the 492 direction of the stimulus at the cued location was changed and the monkey was rewarded for 493 making a saccade to the changed stimulus within $500 \mathrm{~ms}$. As with the MT-SC data, stimulus 494 presentations during which the monkey made a micro-saccade were excluded from analysis, all 495 stimulus presentations after the first and before the orientation change were analyzed, and the 
presentations were sampled such that they were equal in the two attention conditions. Each session yielded 97-1469 (mean 583) presentations for each attention condition.

\section{QUANTIFICATION AND STATISTICAL ANALYSIS}

\section{$500 \quad$ Subsampling}

501 To test whether attention affects prediction of neural responses within and across areas, we first

502 sought to check whether or not the number of recorded neurons and trials across the two

503 attention conditions in the datasets were sufficient for reasonable regression performance. We

504 used a linear model of the form $Y=X B$ where $X$ and $Y$ are matrices of $t x n$ and $t x m$ dimensions

505 and $B$ is the weight matrix of dimensions $n \times m$ (here, $t$ is the number of stimulus presentations in

506 a session, $m$ and $n$ are the numbers of neurons in the two areas). We found the ordinary least-

507 squares solution for B by minimizing the squared prediction error as $B=\left(X^{T} X\right)^{-1} X^{T} Y$. We sampled

$508 \quad N$ MT neurons (where $N$ went from 1 to the total number of recorded MT neurons) without

509 replacement and used ridge regression predict SC responses. We did this subsampling 100 times

510 for each $N$. For ridge regression, we chose the value of the regularization parameter $(\lambda)$ using 10-

511 fold cross-validation. The reported prediction accuracy corresponds to the largest $\lambda$ for which

512 mean performance (across folds) was within one SEM of the best performance. We also used the

513 full MT recorded population to predict the responses of subsets of $N$ SC neurons (where $N$ went

514 from 1 to the total number of recorded SC neurons) using the same method.

\section{Noise correlations}

517 The spike count correlation $\left(\mathrm{r}_{\mathrm{SC}}\right)$ was calculated as the correlation coefficient between the

518 responses of the two units to repeated presentations of the same stimulus. Z-scoring responses

519 before calculating noise correlations did not qualitatively change the comparisons between noise

520 correlations and local or shared dimensionality or prediction accuracy. In Figure S1, noise

521 correlations are computed for each pair in a session using all stimulus presentations in every trial

522 (except the first), and then pooled across sessions and monkeys to yield 3315 pairs in MT, 3975

523 pairs in SC, and 6934 pairs across MT and SC. In Figure S3, noise correlations are computed as

524 above and then averaged for each session. 
527 To find the effect of attention on the ability of MT responses to predict SC responses and vice

528 versa, we used the same linear model described above using ridge regression. This model is referred to as the full regression model in the text. To assess whether the SC activity can be

530 predicted using a subset of MT population response dimensions (in other words, a subspace of

531 MT activity), we used reduced-rank regression (RRR). The exact description and formulation of

532 RRR can be found in (Semedo et al., 2019). Briefly, RRR constrains the weight matrix B to be of

533 a given rank and is solved using singular value decomposition:

$$
Y_{R R R}=X B_{R R R}=X B_{O L S} V V^{T}=X B V^{T}
$$

535 where $B_{O L S}$ is the coefficient matrix for the ordinary least-square solution, $B_{R R R}$ is the coefficient 536 matrix for the RRR solution, $V$ is a matrix whose columns contain the top principal components 537 of the optimal linear predictor $Y_{O L S}=X B_{O L S}$. The columns of $B$ define which dimensions of $X$ are 538 used for generating the prediction i.e., the predictive dimensions. As with the ridge regression 539 solution above, we used 10-fold cross-validation and found the smallest number of dimensions 540 for which predictive performance was within one SEM of the peak performance.

\section{Cross-condition, cross-validated regression}

543 To assess the effect of attention on the structure of the shared subspace between interaction

544 populations of neurons, we calculated how well the regression weight matrix for one condition

545 (attend in, say) predicted the responses of the target population in the opposite condition (attend 546 out). In the first analysis, we simply used the cross-validated optimum number of dimensions to 547 obtain a weight matrix in one condition and tested it against the trials of the other condition. The 548 results of this method are depicted in Figure S7a-d. The accuracy of the inter-areal interaction 549 dropped significantly but the accuracy of the intra-areal interaction was not affected. To assess 550 whether this was a result of a linear scaling of the weight matrix across conditions due to non551 stationarities or other task/stimulus independent factors, we projected the response of the source 552 population using the weight matrix of the opposite condition before performing RR regression to 553 obtain the prediction. This was cross-validated in the following way described in pseudo-code

554 (for the MT $\rightarrow$ SC interaction, for the attend out trials using the attend out vs attend in models, 555 but we followed the same process for all potential permutations of conditions and areas).

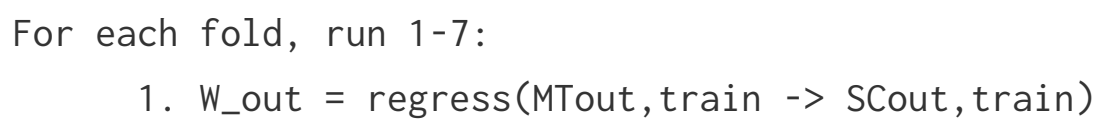


4. MTout, train' $=\operatorname{project}($ MTout, train, W_in)

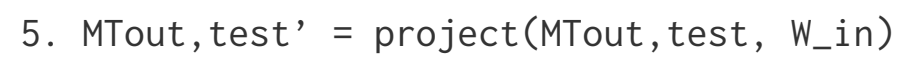
attendOut_NSE_within = NSE (SCout, testPred, SCout, test) ratio = attendOut_NSE_cross/attendOut_NSE_within

The ratio thus obtained was a cross-validated measure of how well the attend out weight matrix activity projected through the attend in weight matrix (W_in) first. We ran this for 10-folds for each random split of each population (described above) and evaluated the ratio of the normalized square error of prediction using both the matrices. This ratio is a quantitative measure of how well the cross-condition weight matrix performs relative to the within-condition weight matrix

\section{Factor Analysis}

579 We used factor analysis (FA) to assess the dimensionality of neural activity within an area. FA is 580 a static dimensionality reduction technique that does not assume the same noise variance for all recorded neurons and calculates the dimensions of greatest covariance (instead of variance). As with RRR, the details of this analysis can be found in previous publications (Everitt, 1984;

583 Semedo et al., 2014; Yu et al., 2009). We followed the same steps as previously published work

584 to estimate the dimensionality: (1) we found the number of dimensions $m_{\text {peak }}$ that maximized the 585 cross-validated log-likelihood of the observed residuals; (2) we fitted a FA model with $m_{\text {peak }}$ 586 dimensions and chose $m$, using the eigenvalue decomposition, as the smallest dimensionality that 587 captured $95 \%$ of the variance in the shared covariance matrix. These population dimensions $(m)$ 588 and predictive dimensions as determined from RRR are determined by different techniques and therefore, wherever applicable, we have used these techniques to evaluate only the change of 
590 dimensionality (private or shared) between the two attention conditions instead of comparing 591 absolute values. 


\section{References}

593 Ardid, S., Wang, X.-J., and Compte, A. (2007). An Integrated Microcircuit Model of Attentional

594 Processing in the Neocortex. J. Neurosci. 27, 8486-8495.

595 Azouz, R., and Gray, C.M. (2003). Adaptive Coincidence Detection and Dynamic Gain Control

596 in Visual Cortical Neurons In Vivo. Neuron 37, 513-523.

597 Bair, W., Zohary, E., and Newsome, W.T. (2001). Correlated firing in macaque visual area MT: 598 time scales and relationship to behavior. J. Neurosci. 21, 1676-1697.

599 Baruni, J.K., Lau, B., and Salzman, C.D. (2015). Reward expectation differentially modulates

600 attentional behavior and activity in visual area V4. Nat. Neurosci. 18, 1656-1663.

601 Benevento, L.A., and Yoshida, K. (1981). The afferent and efferent organization of the lateral 602 geniculo-prestriate pathways in the macaque monkey. J. Comp. Neurol. 203, 455-474.

603 Bichot, N.P., Rossi, A.F., and Desimone, R. (2005). Parallel and Serial Neural Mechanisms for 604 Visual Search in Macaque Area V4. Science 308, 529-534.

605 Bosman, C.A., Schoffelen, J.-M., Brunet, N., Oostenveld, R., Bastos, A.M., Womelsdorf, T., 606 Rubehn, B., Stieglitz, T., De Weerd, P., and Fries, P. (2012). Attentional stimulus selection 607 through selective synchronization between monkey visual areas. Neuron 75, 875-888.

608 Boynton, G.M. (2009). A framework for describing the effects of attention on visual responses.

609 Vision Res. 49, 1129-1143.

610 Brainard, D.H. (1997). The Psychophysics Toolbox. Spat. Vis. 10, 433-436.

611 Briggs, F., Mangun, G.R., and Usrey, W.M. (2013). Attention enhances synaptic efficacy and the 612 signal-to-noise ratio in neural circuits. Nature 499, 476-480.

613 Brunel, N., and Wang, X.-J. (2001). Effects of Neuromodulation in a Cortical Network Model of 614 Object Working Memory Dominated by Recurrent Inhibition. J. Comput. Neurosci. 11, 63-85.

615 Buffalo, E.A., Fries, P., Landman, R., Buschman, T.J., and Desimone, R. (2011). Laminar

616 differences in gamma and alpha coherence in the ventral stream. Proc. Natl. Acad. Sci. 108, $617 \quad 11262-11267$.

618 Buia, C.I., and Tiesinga, P.H. (2008). Role of Interneuron Diversity in the Cortical Microcircuit 619 for Attention. J. Neurophysiol. 99, 2158-2182.

620 Buschman, T.J., and Miller, E.K. (2007). Top-Down Versus Bottom-Up Control of Attention in 621 the Prefrontal and Posterior Parietal Cortices. Science 315, 1860-1862.

622 Cardin, J.A., Carlén, M., Meletis, K., Knoblich, U., Zhang, F., Deisseroth, K., Tsai, L.-H., and 623 Moore, C.I. (2009). Driving fast-spiking cells induces gamma rhythm and controls sensory

624 responses. Nature 459, 663-667. 
625 Carrasco, M. (2011). Visual attention: The past 25 years. Vision Res. 51, 1484-1525.

626 Cohen, M.R., and Kohn, A. (2011). Measuring and interpreting neuronal correlations. Nat.

627 Neurosci. 14, 811-819.

628 Cohen, M.R., and Maunsell, J.H.R. (2009). Attention improves performance primarily by

629 reducing interneuronal correlations. Nat. Neurosci. 12, 1594-1600.

630 Cohen, M.R., and Maunsell, J.H.R. (2011). Using neuronal populations to study the mechanisms 631 underlying spatial and feature attention. Neuron 70, 1192-1204.

632 Cowley, B.R., Smith, M.A., Kohn, A., and Yu, B.M. (2016). Stimulus-Driven Population

633 Activity Patterns in Macaque Primary Visual Cortex. PLOS Comput. Biol. 12, e1005185.

634 Cowley, B.R., Snyder, A.C., Acar, K., Williamson, R.C., Yu, B.M., and Smith, M.A. (2020).

635 Slow Drift of Neural Activity as a Signature of Impulsivity in Macaque Visual and Prefrontal

636 Cortex. Neuron 108, 551-567.e8.

637 Cunningham, J.P., and Yu, B.M. (2014). Dimensionality reduction for large-scale neural

638 recordings. Nat. Neurosci. 17, 1500-1509.

639 Dagnino, B., Gariel-Mathis, M.-A., and Roelfsema, P.R. (2014). Microstimulation of area V4

640 has little effect on spatial attention and on perception of phosphenes evoked in area V1. J.

641 Neurophysiol. 113, 730-739.

642 Deco, G., and Thiele, A. (2011). Cholinergic control of cortical network interactions enables

643 feedback-mediated attentional modulation. Eur. J. Neurosci. 34, 146-157.

644 Desimone, R., and Duncan, J. (1995). Neural Mechanisms of Selective Visual Attention. Annu.

645 Rev. Neurosci. 18, 193-222.

646 Driver, J. (2001). A selective review of selective attention research from the past century. Br. J. 647 Psychol. 92, 53-78.

648 Ecker, A.S., Denfield, G.H., Bethge, M., and Tolias, A.S. (2016). On the Structure of Neuronal 649 Population Activity under Fluctuations in Attentional State. J. Neurosci. 36, 1775-1789.

650 Egeth, H.E., and Yantis, S. (1997). VISUAL ATTENTION: Control, Representation, and Time 651 Course. Annu. Rev. Psychol. 48, 269-297.

652 Elsayed, G.F., and Cunningham, J.P. (2017). Structure in neural population recordings: an 653 expected byproduct of simpler phenomena? Nat. Neurosci. 20, 1310-1318.

654 Elsayed, G.F., Lara, A.H., Kaufman, M.T., Churchland, M.M., and Cunningham, J.P. (2016).

655 Reorganization between preparatory and movement population responses in motor cortex. Nat.

656 Commun. 7, 13239. 
Everitt, B.S. (1984). Maximum Likelihood Estimation of the Parameters in a Mixture of Two Univariate Normal Distributions; a Comparison of Different Algorithms. J. R. Stat. Soc. Ser.

659 Stat. 33, 205-215.

660 Fries, P. (2015). Rhythms for Cognition: Communication through Coherence. Neuron 88, 220235.

662 Fries, W. (1984). Cortical projections to the superior colliculus in the macaque monkey: A retrograde study using horseradish peroxidase. J. Comp. Neurol. 230, 55-76.

664 Fries, W. (1985). Inputs from motor and premotor cortex to the superior colliculus of the 665 macaque monkey. Behav. Brain Res. 18, 95-105.

Fries, P., Reynolds, J.H., Rorie, A.E., and Desimone, R. (2001). Modulation of oscillatory neuronal synchronization by selective visual attention. Science 291, 1560-1563.

668 Fu, Y., Tucciarone, J.M., Espinosa, J.S., Sheng, N., Darcy, D.P., Nicoll, R.A., Huang, Z.J., and 669 Stryker, M.P. (2014). A Cortical Circuit for Gain Control by Behavioral State. Cell 156, 11396701152.

671 Gilbert, C.D., and Sigman, M. (2007). Brain States: Top-Down Influences in Sensory 672 Processing. Neuron 54, 677-696.

673 Goldberg, M.E., and Wurtz, R.H. (1972). Activity of superior colliculus in behaving monkey. II. 674 Effect of attention on neuronal responses. J. Neurophysiol. 35, 560-574.

675 Golub, M.D., Chase, S.M., Batista, A.P., and Yu, B.M. (2016). Brain-computer interfaces for 676 dissecting cognitive processes underlying sensorimotor control. Curr. Opin. Neurobiol. 37, 5367758.

678 Gregoriou, G.G., Gotts, S.J., Zhou, H., and Desimone, R. (2009). High-frequency, long-range 679 coupling between prefrontal and visual cortex during attention. Science 324, 1207-1210.

680 Gregoriou, G.G., Rossi, A.F., Ungerleider, L.G., and Desimone, R. (2014). Lesions of prefrontal 681 cortex reduce attentional modulation of neuronal responses and synchrony in V4. Nat. Neurosci. $682 \quad 17,1003-1011$.

683 Herrero, J.L., Roberts, M.J., Delicato, L.S., Gieselmann, M.A., Dayan, P., and Thiele, A. (2008). 684 Acetylcholine contributes through muscarinic receptors to attentional modulation in V1. Nature $685454,1110-1114$.

686 Herrero, J.L., Gieselmann, M.A., Sanayei, M., and Thiele, A. (2013). Attention-Induced 687 Variance and Noise Correlation Reduction in Macaque V1 Is Mediated by NMDA Receptors. 688 Neuron 78, 729-739.

689 Huang, C., Ruff, D.A., Pyle, R., Rosenbaum, R., Cohen, M.R., and Doiron, B. (2019). Circuit 690 Models of Low-Dimensional Shared Variability in Cortical Networks. Neuron 101, 337-348.e4. 
691 Ignashchenkova, A., Dicke, P.W., Haarmeier, T., and Thier, P. (2004). Neuron-specific

692 contribution of the superior colliculus to overt and covert shifts of attention. Nat. Neurosci. 7 ,

$69356-64$.

694 Indovina, I., and Macaluso, E. (2004). Occipital-parietal interactions during shifts of exogenous

695 visuospatial attention: trial-dependent changes of effective connectivity. Magn. Reson. Imaging

$69622,1477-1486$.

697 Jazayeri, M., and Afraz, A. (2017). Navigating the Neural Space in Search of the Neural Code.

698 Neuron 93, 1003-1014.

699 Kanashiro, T., Ocker, G.K., Cohen, M.R., and Doiron, B. (2017). Attentional modulation of

700 neuronal variability in circuit models of cortex. ELife 6, e23978.

701 Kanitscheider, I., Coen-Cagli, R., and Pouget, A. (2015). Origin of information-limiting noise

702 correlations. Proc. Natl. Acad. Sci. 112, E6973-E6982.

703 Karnani, M.M., Jackson, J., Ayzenshtat, I., Sichani, A.H., Manoocheri, K., Kim, S., and Yuste, 704 R. (2016). Opening Holes in the Blanket of Inhibition: Localized Lateral Disinhibition by VIP

705 Interneurons. J. Neurosci. 36, 3471-3480.

Kaufman, M.T., Churchland, M.M., Ryu, S.I., and Shenoy, K.V. (2014). Cortical activity in the null space: permitting preparation without movement. Nat. Neurosci. 17, 440-448.

Kiani, R., Esteky, H., Mirpour, K., and Tanaka, K. (2007). Object Category Structure in

710 Neurophysiol. 97, 4296-4309.

711 Klink, P.C., Dagnino, B., Gariel-Mathis, M.-A., and Roelfsema, P.R. (2017). Distinct

712 Feedforward and Feedback Effects of Microstimulation in Visual Cortex Reveal Neural

713 Mechanisms of Texture Segregation. Neuron 95, 209-220.e3.

714 Kohn, A., Coen-Cagli, R., Kanitscheider, I., and Pouget, A. (2016a). Correlations and neuronal 715 population information. Annu. Rev. Neurosci. 39, 237-256.

716 Kohn, A., Coen-Cagli, R., Kanitscheider, I., and Pouget, A. (2016b). Correlations and Neuronal

717 Population Information. Annu. Rev. Neurosci. 39, 237-256.

718 Krauzlis, R.J., Lovejoy, L.P., and Zénon, A. (2013). Superior Colliculus and Visual Spatial 719 Attention. Annu. Rev. Neurosci. 36, 165-182.

720 Kuchibhotla, K.V., Gill, J.V., Lindsay, G.W., Papadoyannis, E.S., Field, R.E., Sten, T.A.H., 721 Miller, K.D., and Froemke, R.C. (2017). Parallel processing by cortical inhibition enables 722 context-dependent behavior. Nat. Neurosci. 20, 62-71.

723 Lakatos, P., Karmos, G., Mehta, A.D., Ulbert, I., and Schroeder, C.E. (2008). Entrainment of 724 Neuronal Oscillations as a Mechanism of Attentional Selection. Science 320, 110-113. 
Lavie, N. (2010). Attention, Distraction, and Cognitive Control Under Load. Curr. Dir. Psychol. Sci. 19, 143-148.

727 Lock, T.M., Baizer, J.S., and Bender, D.B. (2003). Distribution of corticotectal cells in macaque. 728 Exp. Brain Res. 151, 455-470.

Luo, T.Z., and Maunsell, J.H.R. (2015). Neuronal Modulations in Visual Cortex Are Associated with Only One of Multiple Components of Attention. Neuron 86, 1182-1188.

731 Lyon, D.C., Nassi, J.J., and Callaway, E.M. (2010). A Disynaptic Relay from Superior

732 Colliculus to Dorsal Stream Visual Cortex in Macaque Monkey. Neuron 65, 270-279.

733 Machens, C.K., Romo, R., and Brody, C.D. (2005). Flexible Control of Mutual Inhibition: A

734 Neural Model of Two-Interval Discrimination. Science 307, 1121-1124.

735 Maunsell, J.H.R. (2015). Neuronal Mechanisms of Visual Attention. Annu. Rev. Vis. Sci. 1, 736 373-391.

737 Mayo, J.P., and Maunsell, J.H.R. (2016). Graded Neuronal Modulations Related to Visual 738 Spatial Attention. J. Neurosci. 36, 5353-5361.

739 Miller, E.K., and Buschman, T.J. (2013). Cortical circuits for the control of attention. Curr. Opin.

740 Neurobiol. 23, 216-222.

741 Miri, A., Warriner, C.L., Seely, J.S., Elsayed, G.F., Cunningham, J.P., Churchland, M.M., and 742 Jessell, T.M. (2017). Behaviorally Selective Engagement of Short-Latency Effector Pathways by 743 Motor Cortex. Neuron 95, 683-696.e11.

744 Mitchell, J.F., Sundberg, K.A., and Reynolds, J.H. (2007). Differential Attention-Dependent 745 Response Modulation across Cell Classes in Macaque Visual Area V4. Neuron 55, 131-141.

746 Mitchell, J.F., Sundberg, K.A., and Reynolds, J.H. (2009). Spatial attention decorrelates intrinsic 747 activity fluctuations in macaque area V4. Neuron 63,879-888.

748 Moore, T., and Armstrong, K.M. (2003). Selective gating of visual signals by microstimulation 749 of frontal cortex. Nature 421, 370-373.

750 Moore, T., and Zirnsak, M. (2017). Neural Mechanisms of Selective Visual Attention. Annu.

751 Rev. Psychol. 68, 47-72.

752 Morcos, A.S., and Harvey, C.D. (2016). History-dependent variability in population dynamics 753 during evidence accumulation in cortex. Nat. Neurosci. 19, 1672-1681.

754 Moreno-Bote, R., Beck, J., Kanitscheider, I., Pitkow, X., Latham, P., and Pouget, A. (2014).

755 Information-limiting correlations. Nat. Neurosci. 17, 1410-1417.

756 Nandy, A.S., Nassi, J.J., and Reynolds, J.H. (2017). Laminar Organization of Attentional

757 Modulation in Macaque Visual Area V4. Neuron 93, 235-246. 
Navalpakkam, V., and Itti, L. (2005). Modeling the influence of task on attention. Vision Res. $45,205-231$.

Ni, A.M., Ruff, D.A., Alberts, J.J., Symmonds, J., and Cohen, M.R. (2018). Learning and attention reveal a general relationship between population activity and behavior. Science 359 ,

$762 \quad 463-465$.

Nienborg, H., and Cumming, B. (2010). Correlations between the activity of sensory neurons and behavior: how much do they tell us about a neuron's causality? Curr. Opin. Neurobiol. 20, $765376-381$.

Nienborg, H., R. Cohen, M., and Cumming, B.G. (2012). Decision-Related Activity in Sensory

Oemisch, M., Westendorff, S., Everling, S., and Womelsdorf, T. (2015). Interareal Spike-Train Correlations of Anterior Cingulate and Dorsal Prefrontal Cortex during Attention Shifts. J.

770 Neurosci. 35, 13076-13089.

771 Ozaki, T.J. (2011). Frontal-to-Parietal Top-Down Causal Streams along the Dorsal Attention

772 Network Exclusively Mediate Voluntary Orienting of Attention. PLOS ONE 6, e20079.

773 Pandarinath, C., O’Shea, D.J., Collins, J., Jozefowicz, R., Stavisky, S.D., Kao, J.C., Trautmann, 774 E.M., Kaufman, M.T., Ryu, S.I., Hochberg, L.R., et al. (2018). Inferring single-trial neural 775 population dynamics using sequential auto-encoders. Nat. Methods 15, 805-815.

776 Parker, A.J., and Newsome, W.T. (1998). SENSE AND THE SINGLE NEURON: Probing the 777 Physiology of Perception. Annu. Rev. Neurosci. 21, 227-277.

778 Peelen, M.V., and Kastner, S. (2014). Attention in the real world: toward understanding its 779 neural basis. Trends Cogn. Sci. 18, 242-250.

780 Pitkow, X., and Angelaki, D.E. (2017). Inference in the Brain: Statistics Flowing in Redundant 781 Population Codes. Neuron 94, 943-953.

782 Pooresmaeili, A., Poort, J., and Roelfsema, P.R. (2014). Simultaneous selection by object-based 783 attention in visual and frontal cortex. Proc. Natl. Acad. Sci. 111, 6467-6472.

784 Recanzone, G.H., and Wurtz, R.H. (2000). Effects of Attention on MT and MST Neuronal 785 Activity During Pursuit Initiation. J. Neurophysiol. 83, 777-790.

786 Reynolds, J.H., and Chelazzi, L. (2004). Attentional Modulation of Visual Processing. Annu. 787 Rev. Neurosci. 27, 611-647.

788 Reynolds, J.H., and Heeger, D.J. (2009). The Normalization Model of Attention. Neuron 61, 789 168-185. 
Roberts, M.J., Zinke, W., Guo, K., Robertson, R., McDonald, J.S., and Thiele, A. (2005). Acetylcholine Dynamically Controls Spatial Integration in Marmoset Primary Visual Cortex. J. Neurophysiol. 93, 2062-2072.

Rodman, H.R., Gross, C.G., and Albright, T.D. (1990). Afferent basis of visual response properties in area MT of the macaque. II. Effects of superior colliculus removal. J. Neurosci. 10, 795 1154-1164.

Rossi, S., Huang, S., Furtak, S.C., Belliveau, J.W., and Ahveninen, J. (2014). Functional connectivity of dorsal and ventral frontoparietal seed regions during auditory orienting. Brain Res. 1583, 159-168.

Rubin, D.B., Van Hooser, S.D., and Miller, K.D. (2015). The Stabilized Supralinear Network: A Unifying Circuit Motif Underlying Multi-Input Integration in Sensory Cortex. Neuron 85, 402801417.

802 Ruff, D.A., and Cohen, M.R. (2014a). Attention can either increase or decrease spike count correlations in visual cortex. Nat. Neurosci. 17, 1591-1597.

804 Ruff, D.A., and Cohen, M.R. (2014b). Global cognitive factors modulate correlated response variability between V4 neurons. J. Neurosci. 34, 16408-16416.

Ruff, D.A., and Cohen, M.R. (2016a). Attention Increases Spike Count Correlations between

808 Ruff, D.A., and Cohen, M.R. (2016b). Stimulus Dependence of Correlated Variability across

809 Cortical Areas. J. Neurosci. 36, 7546-7556.

810 Ruff, D.A., and Cohen, M.R. (2017). A normalization model suggests that attention changes the weighting of inputs between visual areas. Proc. Natl. Acad. Sci. 114, E4085-E4094.

812 Ruff, D.A., and Cohen, M.R. (2019). Simultaneous multi-area recordings suggest that attention 813 improves performance by reshaping stimulus representations. Nat. Neurosci. 22, 1669-1676.

814 Ruff, D.A., Alberts, J.J., and Cohen, M.R. (2016). Relating normalization to neuronal 815 populations across cortical areas. J. Neurophysiol. 116, 1375-1386.

816 Ruff, D.A., Ni, A.M., and Cohen, M.R. (2018). Cognition as a Window into Neuronal Population 817 Space. Annu. Rev. Neurosci.41,77-97.

818 Ruff, D.A., Xue, C., Kramer, L.E., Baqai, F., and Cohen, M.R. (2020). Low rank mechanisms 819 underlying flexible visual representations. Proc. Natl. Acad. Sci. 117, 29321-29329.

820 Saalmann, Y.B., Pigarev, I.N., and Vidyasagar, T.R. (2007). Neural Mechanisms of Visual 821 Attention: How Top-Down Feedback Highlights Relevant Locations. Science 316, 1612-1615.

822 Sadtler, P.T., Quick, K.M., Golub, M.D., Chase, S.M., Ryu, S.I., Tyler-Kabara, E.C., Yu, B.M., 823 and Batista, A.P. (2014). Neural constraints on learning. Nature 512, 423-426. 
824 Salinas, E., and Sejnowski, T.J. (2001). Correlated neuronal activity and the flow of neural

825 information. Nat. Rev. Neurosci. 2, 539-550.

826 Saproo, S., and Serences, J.T. (2014). Attention Improves Transfer of Motion Information

827 between V1 and MT. J. Neurosci. 34, 3586-3596.

828 Seidemann, E., and Newsome, W.T. (1999). Effect of Spatial Attention on the Responses of 829 Area MT Neurons. J. Neurophysiol. 81, 1783-1794.

830 Semedo, J., Zandvakili, A., Kohn, A., Machens, C.K., and Yu, B.M. (2014). Extracting Latent

831 Structure From Multiple Interacting Neural Populations. Adv. Neural Inf. Process. Syst. 27.

832 Semedo, J.D., Zandvakili, A., Machens, C.K., Yu, B.M., and Kohn, A. (2019). Cortical Areas

833 Interact through a Communication Subspace. Neuron 102, 249-259.e4.

834 Semedo, J.D., Gokcen, E., Machens, C.K., Kohn, A., and Yu, B.M. (2020). Statistical methods

835 for dissecting interactions between brain areas. Curr. Opin. Neurobiol. 65, 59-69.

836 Semedo, J.D., Jasper, A.I., Zandvakili, A., Aschner, A., Machens, C.K., Kohn, A., and Yu, B.M. 837 (2021). Feedforward and feedback interactions between visual cortical areas use different

838 population activity patterns. BioRxiv 2021.02.08.430346.

839 Silver, R.A. (2010). Neuronal arithmetic. Nat. Rev. Neurosci. 11, 474-489.

840 Stepniewska, I., Qi, H.-X., and Kaas, J.H. (1999). Do superior colliculus projection zones in the 841 inferior pulvinar project to MT in primates? Eur. J. Neurosci. 11, 469-480.

842 Sutherland, M.R., McQuiggan, D.A., Ryan, J.D., and Mather, M. (2017). Perceptual salience 843 does not influence emotional arousal's impairing effects on top-down attention. Emotion 17 , $844700-706$.

845 Umakantha, A., Morina, R., Cowley, B.R., Snyder, A.C., Smith, M.A., and Yu, B.M. (2020).

846 Bridging neuronal correlations and dimensionality reduction. BioRxiv 2020.12.04.383604.

847 Veit, J., Hakim, R., Jadi, M.P., Sejnowski, T.J., and Adesnik, H. (2017). Cortical gamma band 848 synchronization through somatostatin interneurons. Nat. Neurosci. 20,951-959.

849 Verhoef, B.-E., and Maunsell, J.H.R. (2017). Attention-related changes in correlated neuronal 850 activity arise from normalization mechanisms. Nat. Neurosci. 20, 969-977.

851 Womelsdorf, T., and Fries, P. (2007). The role of neuronal synchronization in selective attention. 852 Curr. Opin. Neurobiol. 17, 154-160.

853 Womelsdorf, T., Fries, P., Mitra, P.P., and Desimone, R. (2006a). Gamma-band synchronization 854 in visual cortex predicts speed of change detection. Nature 439, 733-736.

855 Womelsdorf, T., Anton-Erxleben, K., Pieper, F., and Treue, S. (2006b). Dynamic shifts of visual 856 receptive fields in cortical area MT by spatial attention. Nat. Neurosci. 9, 1156-1160. 
857 Yan, Y., Rasch, M.J., Chen, M., Xiang, X., Huang, M., Wu, S., and Li, W. (2014). Perceptual

858 training continuously refines neuronal population codes in primary visual cortex. Nat. Neurosci.

$85917,1380-1387$.

860 Yu, B.M., Cunningham, J.P., Santhanam, G., Ryu, S.I., Shenoy, K.V., and Sahani, M. (2009).

861 Gaussian-Process Factor Analysis for Low-Dimensional Single-Trial Analysis of Neural

862 Population Activity. J. Neurophysiol. 102, 614-635.

863 Zénon, A., and Krauzlis, R.J. (2012). Attention deficits without cortical neuronal deficits. Nature 864 489, 434-437. 


\section{Supplementary Figures}

\section{Figure S1 - related to figure 2:}

(0.5-page width -1 column)
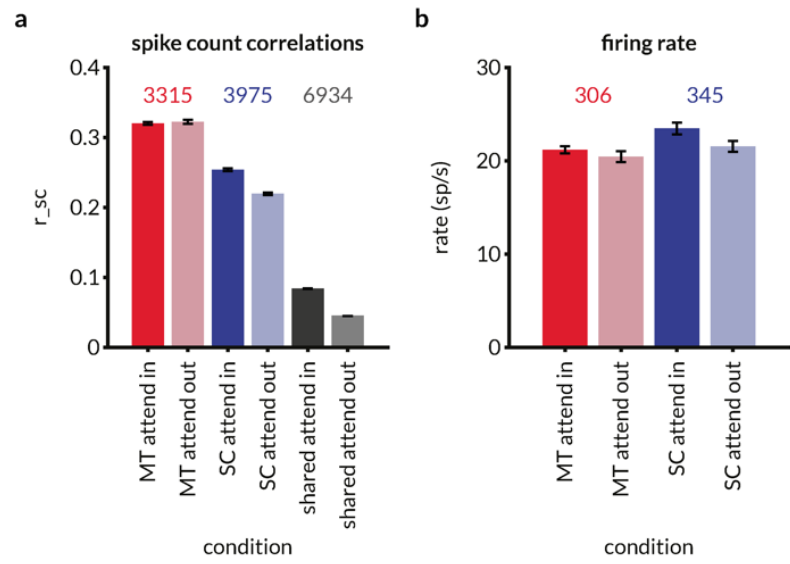

Effect of attention on aggregate noise correlations and firing rates for all neurons and pairs across all recording sessions. Error bars are standard error of the mean. a: Spike count correlations $\left(\mathrm{r}_{\mathrm{SC}}\right)$ for $3315 \mathrm{MT}$ neuron pairs (red), $3975 \mathrm{SC}$ neuron pairs (blue), and 6934 MT-SC pairs (gray) for attend in and attend out conditions. $\mathrm{r}_{\mathrm{SC}}$ was calculated as the Pearson correlation between spike counts during all identical stimulus presentations except the first presentation after the beginning of the trial. Attention increases spike count correlations in SC pairs $\left(\mathrm{p}=2.7 \times 10^{-69}\right.$; Wilcoxon signed rank test) and MT-SC pairs ( $\mathrm{p}=9.1 \times 10^{-224}$; Wilcoxon signed rank test) and has no effect on MT pairs ( $\mathrm{p}=0.8$; Wilcoxon signed rank test). The disparity between these results and previously published results is largely due to the selection of stimulus presentations. Here, we chose all presentations in a trial to increase statistical power in regression and factor analyses, whereas previous publications chose only the stimulus presentation before the orientation change to compare $\mathrm{r}_{\mathrm{SC}}$ with behavioral outcomes.

b: Average firing rate across all presentations for $306 \mathrm{MT}$ neurons (red) and $345 \mathrm{SC}$ neurons (blue). Attention significantly increases firing rates of neurons in both MT ( $\mathrm{p}=8.87 \times 10^{-14}$; Wilcoxon signed rank test) and $\mathrm{SC}\left(\mathrm{p}=5.88 \times 10^{-42}\right.$; Wilcoxon signed rank test). 
Figure S2 - related to figure 4:

(0.75-page width -1.5 column)

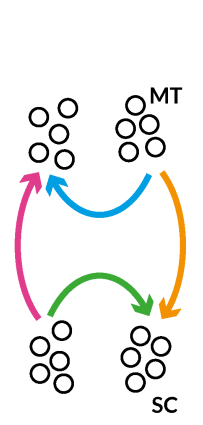

a

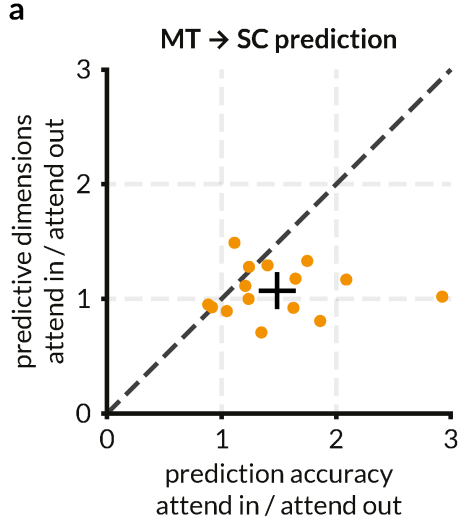

c

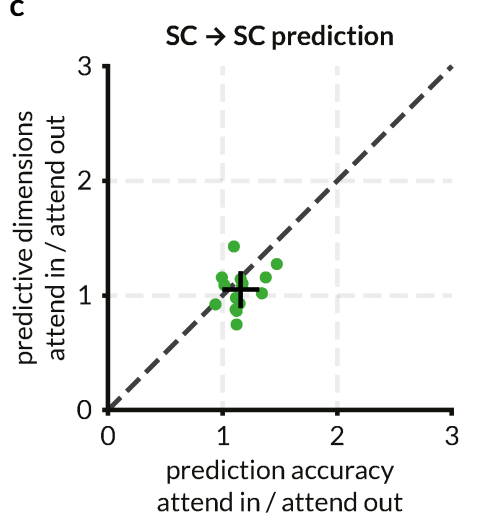

b

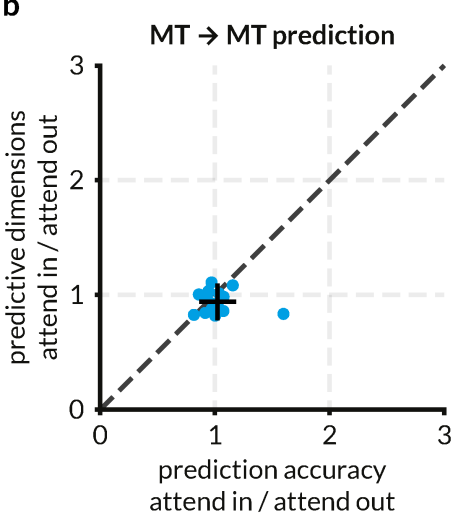

d

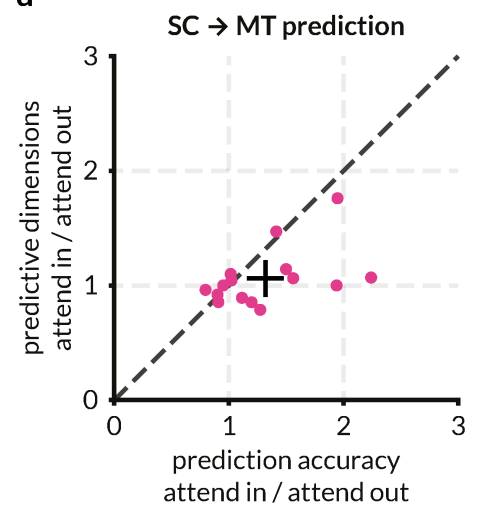

Attention improves prediction accuracy but not predictive dimensions for interareal communication.

Each point represents a recording session, and the color scheme is the same as other figures and redundant with the plot labels. + represents the mean of the points. (same data as Figure $4 \mathrm{c}$ plotted separately for each prediction) a: Prediction accuracy and predictive dimensions presented as ratios

between attend in and attend out conditions for the prediction of SC activity from MT activity. Each dot represents the average prediction accuracy and average predictive dimensions across 100 predictions of a random half of the SC population predicted by a random half of the MT population in that session. Attention increases prediction accuracy of MT $\rightarrow$ SC predictions $(\mathrm{p}=0.0032$; $\mathrm{t}$-test $)$ while having no effect on the number of predictive dimensions.

b: Same as (a) but for MT $\rightarrow$ MT predictions. Each dot represents the average prediction accuracy and average predictive dimensions across 100 predictions of a random half of the MT population predicted by the other half of the same population in that session. Attention has no effect on prediction accuracy or predictive dimensions.

c: Same as (b) but for SC $\rightarrow$ SC predictions. Attention has a small but significant effect on the prediction accuracy $\left(\mathrm{p}=7.9 \times 10^{-4} ; \mathrm{t}\right.$-test) but no effect on predictive dimensions.

d: Same as (a) but for SC $\rightarrow$ MT predictions. Attention increases prediction accuracy of SC $\rightarrow$ MT predictions ( $\mathrm{p}=0.0142$; $\mathrm{t}$-test) while having no effect on the number of predictive dimensions. 
Figure S3 - related to figure 4:

(full page width -2 column)
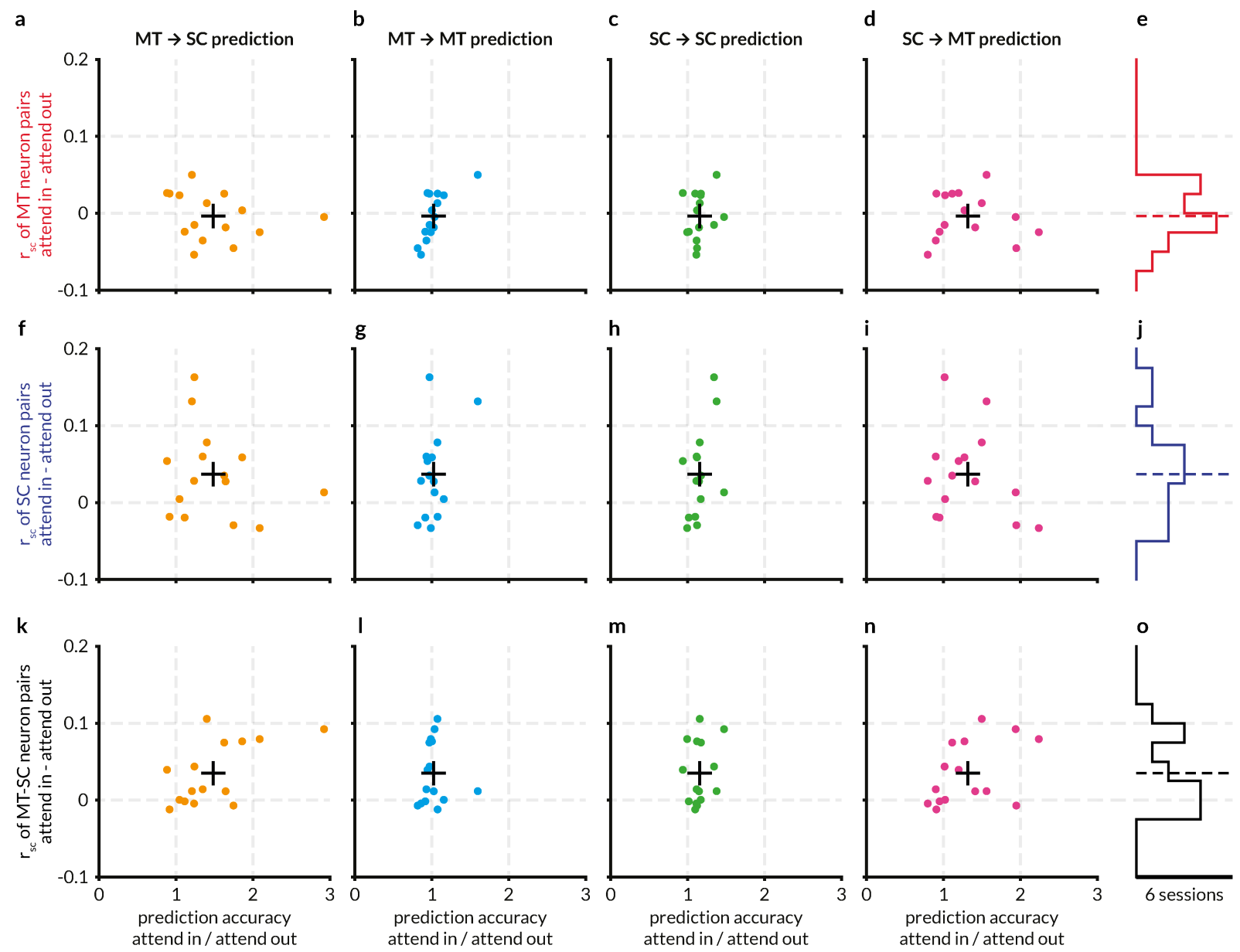

Attention-related changes in spike count correlations do not predict the improvement in communication efficacy across areas. Each panel illustrates how the differences of noise correlations of MT neuron pairs (a-e), SC neuron pairs (f-j), and MT-SC neuron pairs (k-o) between attend in and attend out conditions relate to the ratio of accuracies for within and across area response predictions. Each point represents a recording session, and the color scheme is the same as other figures and redundant with the plot labels. + represents the mean of the points. a: No relationship between the effect of attention on the average accuracy of MT $\rightarrow$ SC predictions for each session and the effect on the average spike count correlations for MT neuron pairs for the same session.

b: Same as (a) for MT $\rightarrow$ MT predictions.

c: Same as (a) for SC $\rightarrow$ SC predictions.

d: Same as (a) for $\mathrm{SC} \rightarrow \mathrm{MT}$ predictions.

e: Histogram of the difference of $r_{S C}$ of MT neuron pairs between the two attention conditions. Dotted line represents the mean of -0.0035 . 
f-g: Same as a-e, but for comparing prediction accuracies with session-wise average spike count correlations for SC neuron pairs. Dotted line in the histogram in $(\mathrm{g})$ represents the mean of 0.0369 .

k-o: Same as a-e, but for comparing prediction accuracies with session-wise average spike count correlations for MT and SC neuron pairs. Dotted line in the histogram in (o) represents the mean of 0.0350 . A weak relationship may be observed in $(\mathrm{k})$ and $(\mathrm{n})$ but the adjusted $\mathrm{r}^{2}$ for linear model fits are low ( 0.303 and 0.145 respectively) and not significant vs constant model. 
Figure S4 - related to figure 4:

(full page width -2 column)
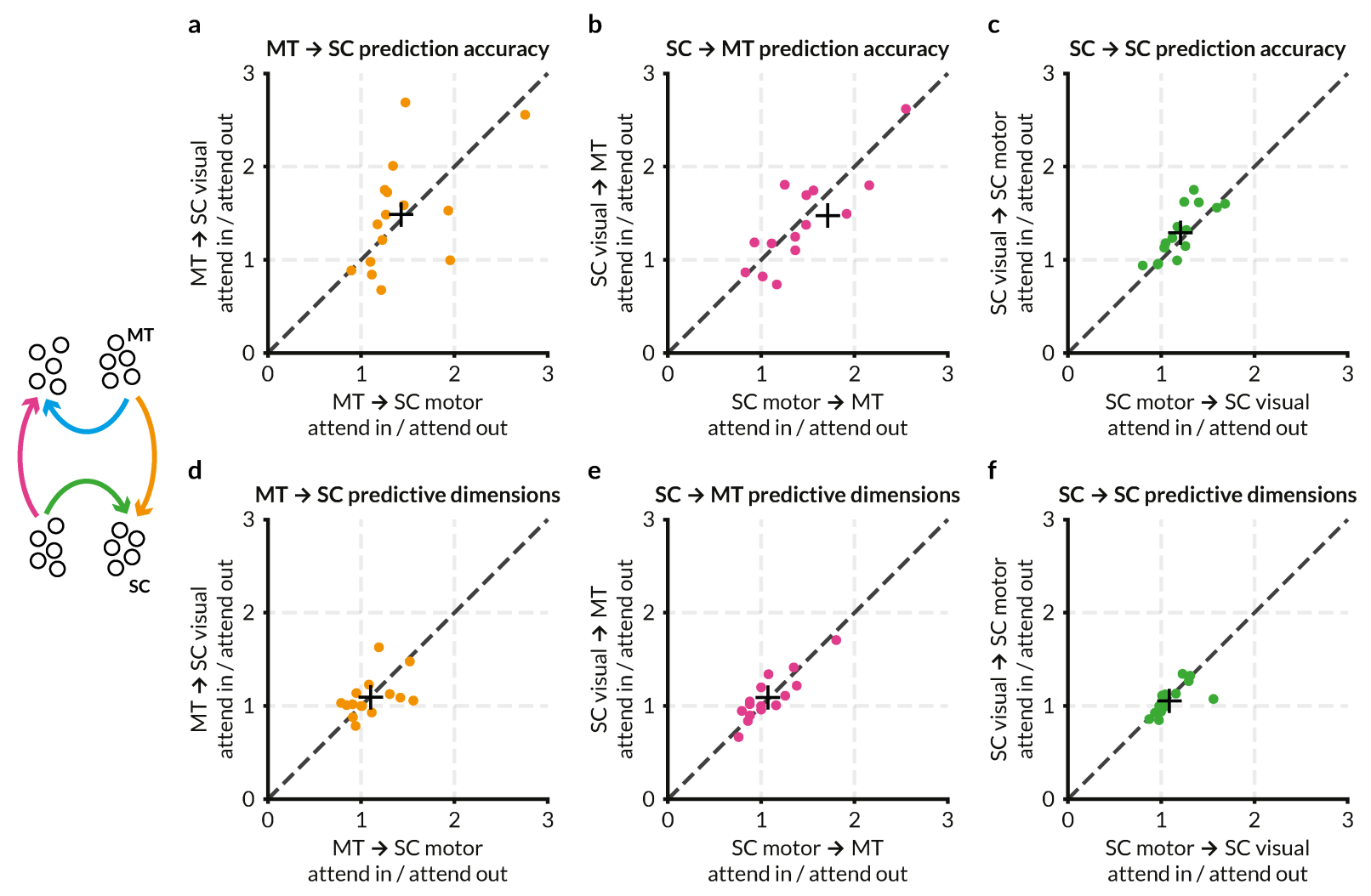

Both oculo-motor and motor neurons in SC contribute similarly to the attention-related improvement in prediction performance between MT and SC. For each session, SC neurons were ordered by an oculo-motor score (described in text and methods) and split evenly into "SC visual" and "SC motor" populations. (Oculo-motor SC neurons are labeled "SC visual" for brevity.) Each point represents a recording session, and the color scheme is the same as other figures and redundant with the plot labels. + represents the mean of the points.

a: Average accuracy of predictions of randomly split SC populations of either oculo-motor neurons or motor neurons from the same population of randomly sampled MT populations presented as a ratio of the two attention conditions. (In each iteration, 50\% of randomly sampled (without replacement) MT neurons were used to predict $50 \%$ of randomly sampled SC neurons from the top half of the oculo-motor index distribution and $50 \%$ of randomly sampled SC neurons from the bottom half of the distribution. So, effectively, only $25 \%$ of the SC neurons were used for predictions in these regressions as compared to $50 \%$ in other analyses.) The prediction accuracy of both oculo-motor SC and motor SC neural activity from MT neuron activity is similarly elevated with attention. Compare with figure $4 \mathrm{c}$ and supplementary figure 4a-a. $(p=0.0031$ for MT $\rightarrow$ SC motor, $p=0.0071$ for MT $\rightarrow$ SC visual, $p=0.52$ for the ratio of the two; one-sample t-test for the ratios) 
b: Same as (a) for SC oculo-motor or motor $\rightarrow$ MT predictions. As with (a), prediction accuracy is similarly enhanced with attention. Compare with figure $4 \mathrm{c}$ and supplementary figure $4 \mathrm{a}-\mathrm{d}$. $(\mathrm{p}=$ 0.0309 for $\mathrm{SC}$ motor $\rightarrow \mathrm{MT}, \mathrm{p}=0.0052$ for $\mathrm{SC}$ visual $\rightarrow \mathrm{MT}, \mathrm{p}=0.456$ for the ratio of the two; one-sample t-test for the ratios)

c: Same as (a) for recurrent connections between SC oculo-motor and SC motor populations. As with (a), prediction accuracy is enhanced with attention. Compare with figure $4 \mathrm{c}$ and supplementary figure 4a-c. ( $p=0.0047$ for SC motor $\rightarrow$ SC visual, $p=0.0013$ for SC visual $\rightarrow$ $\mathrm{SC}$ motor, $\mathrm{p}=0.0495$ for the ratio [SC visual $\rightarrow \mathrm{SC}$ motor] / [SC motor $\rightarrow \mathrm{SC}$ visual]) d: Same as (a) but for the ratio of the average number of predictive dimensions between the two attention conditions for the $\mathrm{MT} \rightarrow \mathrm{SC}$ oculo-motor or SC motor predictions. Attention has no effect on the dimensionality of the shared subspace between MT and SC populations. Compare with figure $4 c$ and supplementary figure $4 a-a$. ( $p>0.05$ for all ratios; $t$-test) e: Same as (b) for the ratio of the average number of predictive dimensions between the two attention conditions for the SC oculo-motor or SC motor predictions $\rightarrow$ MT predictions. Compare with figure $4 \mathrm{c}$ and supplementary figure $4 \mathrm{a}-\mathrm{d}$. ( $\mathrm{p}>0.05$ for all ratios; $\mathrm{t}$-test) f: Same as (c) for the ratio of the average number of predictive dimensions between the two attention conditions for the recurrent connections between the SC oculo-motor and SC motor populations. Compare with figure $4 \mathrm{c}$ and supplementary figure $4 \mathrm{a}-\mathrm{c}$. ( $\mathrm{p}>0.05$ for all ratios; $\mathrm{t}-$ test) 


\section{Figure S5 - related to figure 5:}

(full page width -2 column)

a

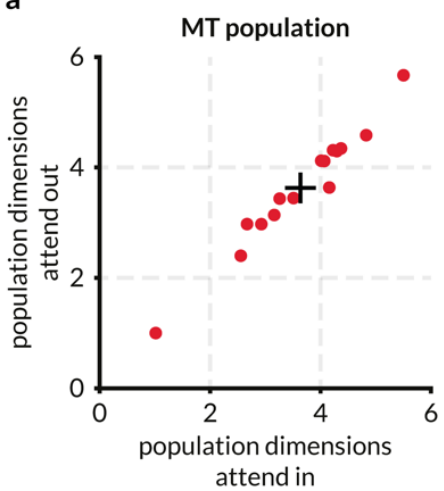

b

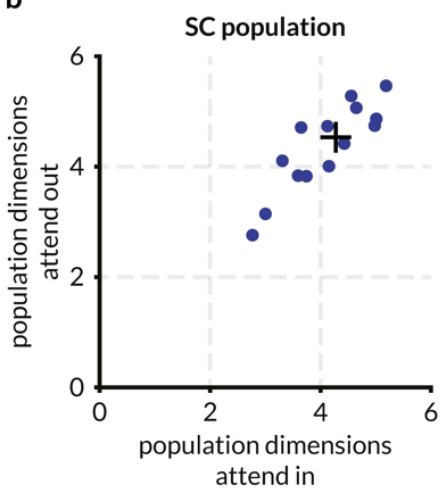

C

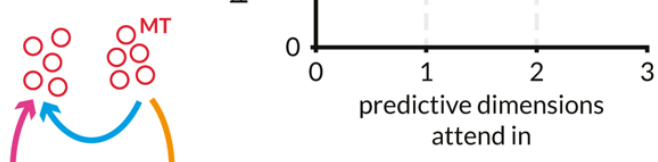

e

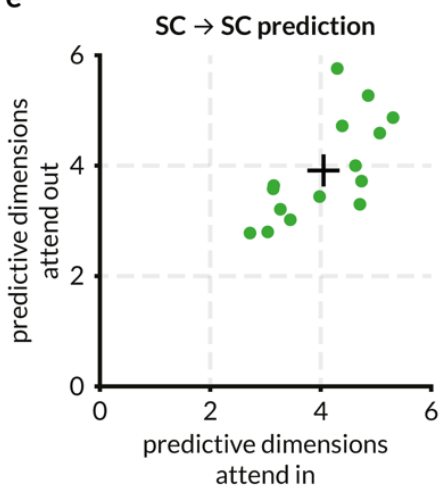

d

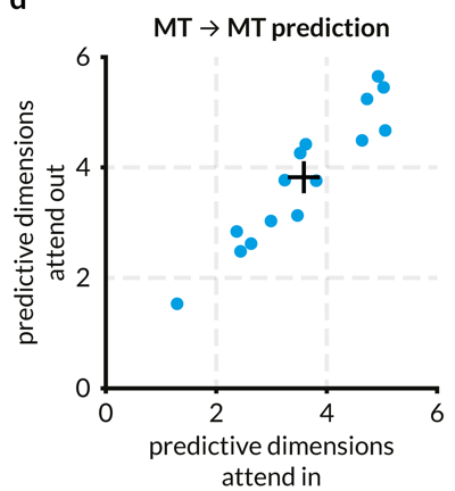

f

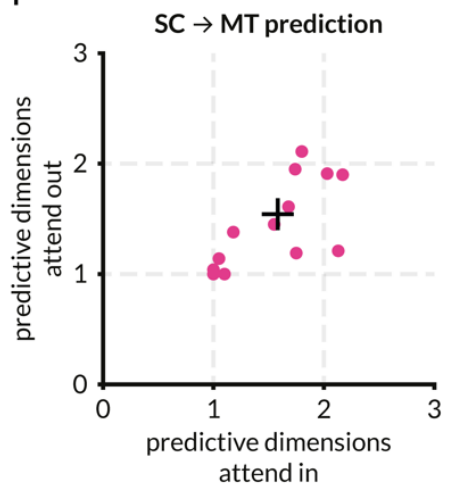

Attention does not alter the dimensionality of the response space in SC or MT, or the dimensionality of the shared communication subspace. Each point represents a recording session, and the color scheme is the same as other figures and redundant with the plot labels. + represents the mean of the points.

a: Attention does not affect the population dimensionality of the MT populations. Each point represents the average number of dimensions (factors) required to explain $95 \%$ of the variance in the MT activity for one session. On average, fluctuations in MT activity are largely restricted to $\sim 3.5$ dimensions.

b: Attention does not affect the population dimensionality of the SC populations. Same as (a) for the SC population. On average, fluctuations in SC activity are largely restricted to $\sim 4.2$ dimensions.

c-f: Attention does not affect the number of dimensions required to optimally predict target activity for any of the four predictions. Same data as figure 4a split into four panels for clarity. 
Figure S6 - related to figure 5:

(full page width -2 column)

a

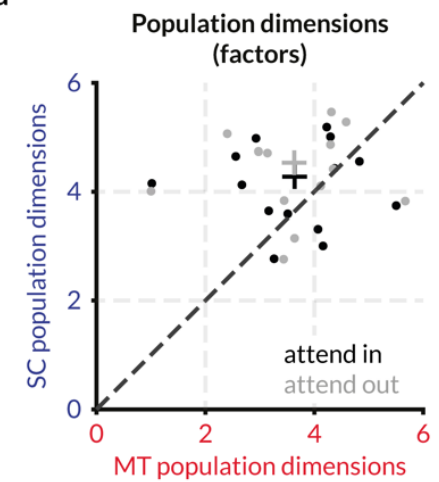

b

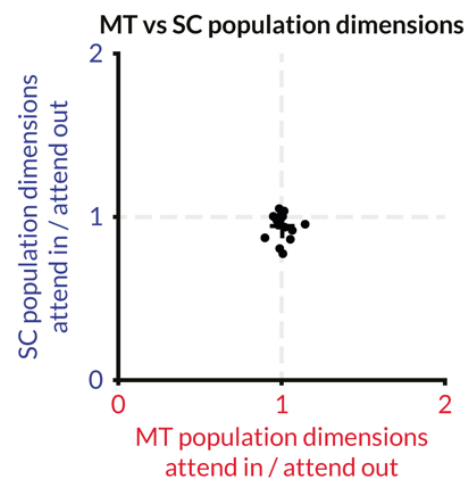

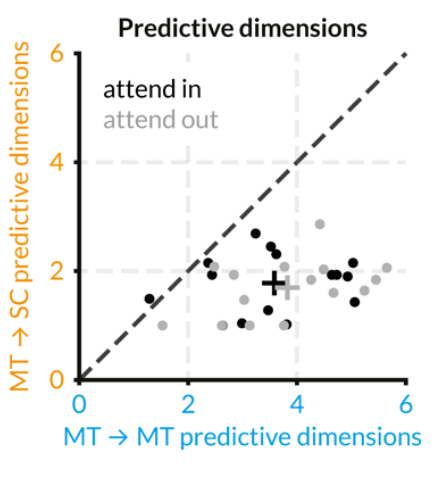

d

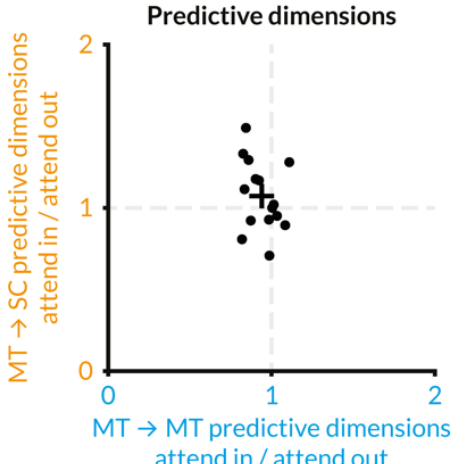

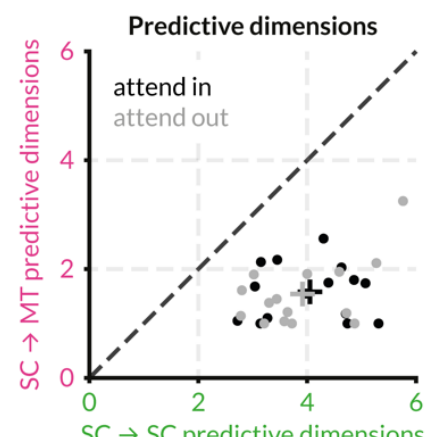

$\mathrm{SC} \rightarrow \mathrm{SC}$ predictive dimensions

f

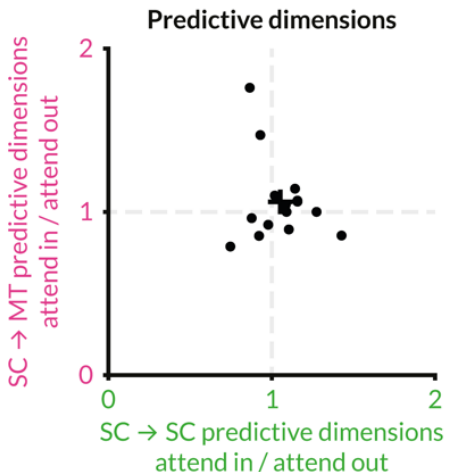

Detailed comparison of attention-related changes in MT and SC population dimensions and predictive dimensions different predictions. Each point represents a recording session, and the color scheme is the same as other figures and redundant with the plot labels. Colored + represents the mean of the corresponding points.

a: Number of population dimensions or factors from factor analysis for the MT and SC populations in each session for attend in and attend out conditions. 95\% of the variance in the MT and SC population activity can be explained with approximately 3.5 and 4.3 dimensions respectively in both attention conditions.

b: Same as (a) expressed as a ratio of population dimensions in attend in and attend out conditions. Attention has no effect on the number of dimensions required to explain $95 \%$ of the variance in activity in this dataset.

c: Number of predictive dimensions that are "shared" between MT and SC (orange axis) vs the number of dimensions that are "private" in MT (blue axis) in the two attention conditions. The number of MT dimensions required to predict SC activity $(\sim 2)$ is lower than the number of MT dimensions required to predict MT activity $(\sim 4)$.

d: Same as (c) expressed as a ratio of predictive dimensions in attend in and attend out conditions. 
e: Same as (c) but for the number of dimensions in SC population activity that is sufficient to explain MT activity. Number of dimensions "shared" between SC and MT $(\sim 2)$ in SC activity is lower than the number of "private" SC dimensions $(\sim 4)$.

f: Same as (e) expressed as a ratio of predictive dimensions in attend in and attend out conditions. 
Figure S7 - related to figure 5:

(0.75-page width -1.5 column)

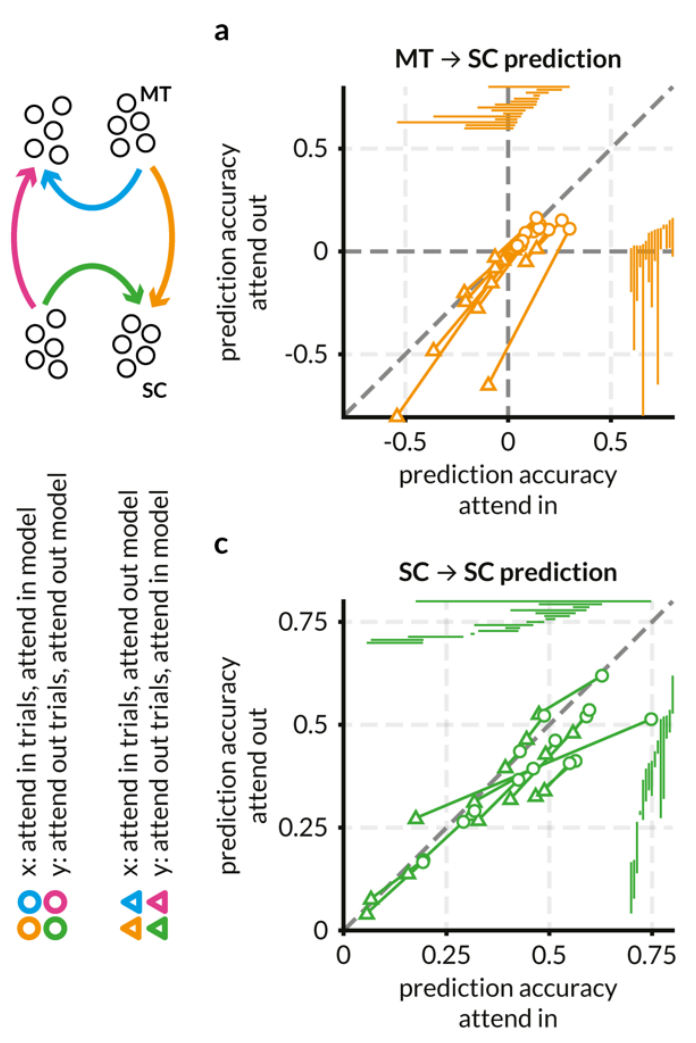

e

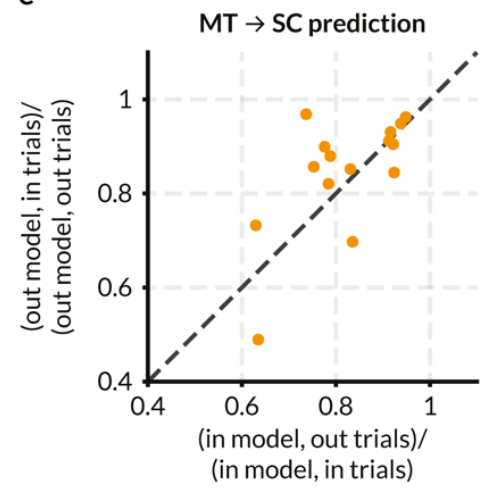

g

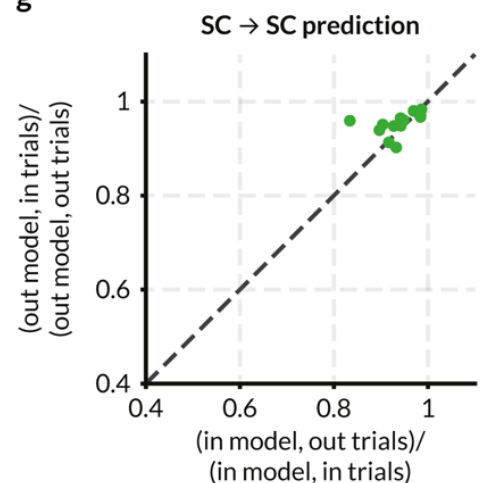

b

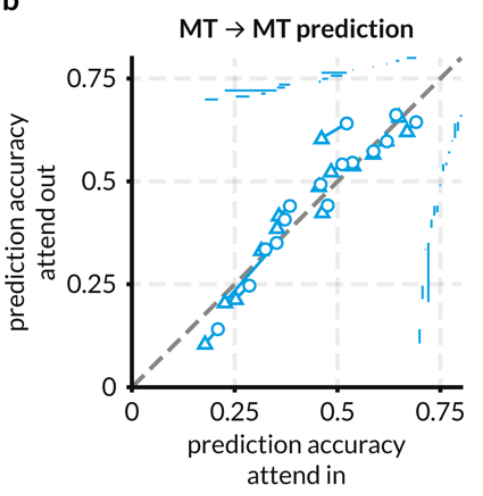

d

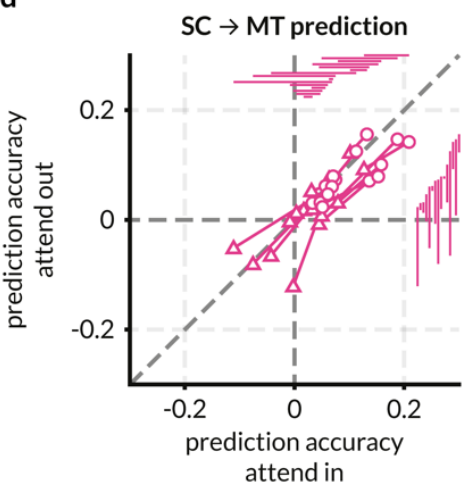

f

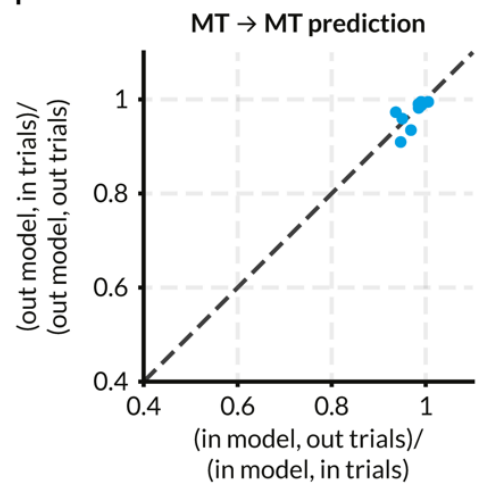

h

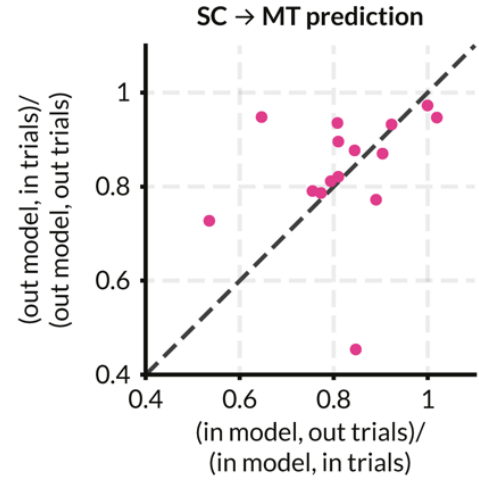

Cross-predicting activity for attend in trials using attend out model and vice versa reveals that the linear subspaces for across area communication are not identical. While the dimensionality of the communication subspace is not affected by attention, it is possible that the structure of the subspace changes while keeping its dimensionality, in turn causing the prediction accuracy to be better. To test this hypothesis, we used the weights of the linear model that corresponded to the optimum number of predictive dimensions in the attend in condition and used it to predict the target responses in the attend out condition and vice versa. We observed a marked drop in performance for cross-prediction for inter-areal communication in 
both directions but not intra-areal communication (a-d). To test whether this drop was due to a linear scaling of the weights across conditions and to cross-validate the cross-predictions, we projected the source activity through the weight matrix of the opposite attention condition and then fit a linear model to the target activity (see Methods for the details of the algorithm) and plotted the cross-validated cross-prediction performance normalized by the cross-validated performance of the true model. We observed a reduction in performance for the inter-areal predictions, albeit milder than earlier estimates (e-f). The intra-areal communication channels remained unaffected. While it may be possible that inter-areal communication indeed utilizes a different assortment of shared dimensions across attention conditions, we assert that these linear methods afford us a partial view of the effect of attention on the communication between areas. Each point represents the mean prediction accuracy of a recording session, and the color scheme is the same as other figures and redundant with the plot labels.

a: We plotted the average cross-prediction accuracy (triangles) for each session and each communication channel across random splits against the true prediction accuracy (circles) i.e., the cross-validated prediction accuracy of the attend in models with the attend in trials etc. The linear model trained to predict SC activity using MT responses in the attend in condition performs significantly ( $\mathrm{p}=2.62 \times 10^{-4}$; Wilcoxon rank sum test) worse when used to predict the $\mathrm{SC}$ responses for trials in the opposite attend out condition; the same is true for the reverse using the attend out model to predict the attend in responses $\left(\mathrm{p}=2.33 \times 10^{-5}\right.$; Wilcoxon rank sum test). Circles represent mean cross-validated prediction accuracy across random splits MT and SC neurons (same points as figure 4a). For each random split, the linear model of the opposite set of trials was used to predict the responses; the mean accuracy this out-of-set prediction across all random splits is represented by the triangles. Each circle-triangle pair is connected by a line and represents the change in prediction performance for a single session. The projections of each line on the cardinal axes are shown on the top and right of the plot, ordered by the prediction accuracy. Out-of-set prediction accuracies are always lower $\left(\mathrm{p}=2.62 \times 10^{-4}\right.$; Wilcoxon rank sum test) and not significantly different from $0(\mathrm{p}=0.07$; t-test), which may mean that the model is unable to do better than guessing the target variance based on the mean of the target population activity (see Semedo et al., 2019 for more details). Both out-of-set models are similarly affected, evident from the consistent slope of the lines. This drastic drop in performance suggests that the shared communication subspace between MT and SC is different across attention conditions. b: Out-of-set mean accuracies for the MT $\rightarrow$ MT prediction are not significantly different $(\mathrm{p}=$ 0.68 for the attend in model and $\mathrm{p}=0.65$ for the attend out model for attend in vs attend out trials; Wilcoxon rank sum test) suggesting not only that attention does not affect prediction performance within MT, but also that the same axes of fluctuations within the MT population activity are used for communication within MT thereby using the same private communication subspace.

c: Same as (b) but for SC $\rightarrow$ SC prediction. The out-of-set prediction is not significantly different ( $p=0.046$ for the attend in model and $p=0.097$ for the attend out model for attend in vs attend out trials; Wilcoxon rank sum test). 
d: Same as (a) but for SC $\rightarrow$ MT predictions. The out-of-set prediction is significantly worse for both the attend in model ( $\mathrm{p}=0.0011$; Wilcoxon rank sum test) and the attend out model ( $\mathrm{p}=$ 0.0016; Wilcoxon rank sum test).

e: To control for the case where the prediction weights across conditions may be linearly scaled and thereby produce significantly worse predictions, the following procedure was used (these steps are for comparing the MT $\rightarrow$ SC attend in weights with the attend out trials, but the same procedure applies for all possible permutations of conditions and populations). The pseudo-code for this cross-validated cross-prediction method can be found in Methods. First, the MT $\rightarrow$ SC prediction weights were found for a set of attend out training trials (W_out) and the SC activity was predicted for the test trials (SCout, testPred). Similarly, the prediction weights for the training set of attend in trials was found (W_in). Then $W_{-}$in was used to project the attend out MT activity for both training and test trials and then used to predict SC activity in the attend out condition for the test trials (SCout, testPredCross). After finding predictions across all folds, the normalized square error was found and compared for the within and across condition predictions. The ratio of the across/within condition prediction for the attend in trials for each session is plotted against the ratio of the across/within condition prediction for the attend out trials. This comparison between these variables demonstrates the ability of the same communication subspace being applied to the trials in the opposite condition and therefore a ratio substantially lower than 1 would indicate that the populations communicate using different subspaces in the different conditions. The cross-prediction accuracy is significantly lower for both attend in and attend out models tested with attend out and attend in trials respectively. f: same as $\mathbf{e}$, but for MT $\rightarrow$ MT interactions. As in $\mathbf{b}$, the performance of the model from the opposite condition does not reduce prediction performance significantly.

g: same as $\mathbf{e}$, but for SC $\rightarrow$ SC interactions. While the cross-prediction accuracy was not significantly different across the two attention conditions in $\mathbf{c}$, the performance of the model was lower in each session. Here, the cross-validated cross-performance shows little difference in the ratio, which provides more evidence for the hypothesis that attention does not alter the dimensionality or structure of the SC-SC communication subspace.

h: same as $\mathbf{e}$, but for SC $\rightarrow$ MT interactions. As in $\mathbf{e}, \mathrm{SC} \rightarrow \mathrm{MT}$ cross-prediction accuracy is significantly lower for both attend in and attend out models tested with attend out and attend in trials respectively. This difference in the structure or the constitution of the communication subspace between MT and SC between attention conditions may be evidence for attention either (a) altering the weights of interareal communication at a fast trial-to-trial timescale by unknown mechanisms, or (b) the inability of linear methods like FA and RRR to describe potentially nonlinear response spaces and the non-linear dynamics of intra- and inter-areal interactions. 